BNL-HET-04/13, LBNL-55325

\title{
Flavor Structure of Warped Extra Dimension Models
}

\author{
Kaustubh Agashe ${ }^{1}$ \\ Department of Physics and Astronomy \\ Johns Hopkins University \\ Baltimore, MD 21218-2686 \\ Gilad Perez ${ }^{2}$ \\ Theoretical Physics Group \\ Lawrence Berkeley National Laboratory \\ Berkeley, CA 94720 \\ and \\ Amarjit Soni ${ }^{3}$ \\ High Energy Theory Group \\ Brookhaven National Laboratory \\ Upton, New York 11973
}

\begin{abstract}
We recently showed, in hep-ph/0406101 that warped extra dimensional models with bulk custodial symmetry and few TeV KK masses lead to striking signals at $B$-factories. In this paper, using a spurion analysis, we systematically study the flavor structure of models that belong to the above class. In particular we find that the profiles of the zero modes, which are similar in all these models, essentially control the underlying flavor structure. This implies that our results are robust and model independent in this class of models. We discuss in detail the origin of the signals in B-physics. We also briefly study other NP signatures that arise in rare $\mathrm{K}$ decays $(K \rightarrow \pi \nu \nu)$, in rare top decays $[t \rightarrow c \gamma(Z$, gluon $)]$ and the possibilty of $\mathrm{CP}$ asymmetries in $D^{0}$ decays to $\mathrm{CP}$ eigenstates such as $K_{S} \pi^{0}$ and others. Finally we demonstrate that with light KK masses, $\sim 3 \mathrm{TeV}$, the above class of models with anarchic $5 D$ Yukawas has a "CP problem" since contributions to the neutron electric dipole moment are roughly 20 times larger than the current experimental bound. Using AdS/CFT correspondence, these extra-dimensional models are dual to a purely $4 D$ strongly coupled conformal Higgs sector thus enhancing their appeal.
\end{abstract}

\footnotetext{
${ }^{1}$ email: kagashe@pha.jhu.edu

${ }^{2}$ email: Gperez@lbl.gov

${ }^{3}$ email: soni@bnl.gov
} 


\section{Introduction}

The Standard Model (SM) has been very successful so far. Almost all of its predictions that have been tested were verified to high precision. Nevertheless, the SM raises the fine tuning problem - which is related to the smallness of the ratio $\Lambda_{\mathrm{EWSB}} / M_{\mathrm{Pl}}$, where $\Lambda_{\mathrm{EWSB}}$ is the electroweak symmetry breaking (EWSB) scale and $M_{\mathrm{Pl}}$ is the Planck mass. The Randall-Sundrum model (RS1), with a warped extra dimension (WED), provides a natural solution to the above problem [1: due to warping, there is exponential hierarchy between the effective cut-off scales of the theory at the two ends of the extra dimension. Thus $\Lambda_{\mathrm{EWSB}}$ is protected due to a low cut-off near the $\mathrm{TeV}$ brane while the high scale of gravity is generated at the other end.

In the original RS1 model the SM fields live on the TeV brane. Consequently, the model cannot solve the flavor puzzle, i.e., why are most of the flavor parameters small and hierarchical. Furthermore, higher dimension operators that induce contribution to FCNC processes are suppressed only by powers of $\Lambda_{\mathrm{EWSB}}$ (the effective cut-off). As well known, however, the cut-off required to suppress $\epsilon_{K}$ and other observables should be higher than $\mathcal{O}(1000) \mathrm{TeV}$. The coefficients of these higher-dimensional operators, which cannot be calculated from first principles, can be in fact small. Consequently, in the original RS1 framework flavor issues are UV sensitive. Similar arguments apply also to constraints from electroweak precision measurements (EWPM) which required cut-off higher than $\mathcal{O}(10) \mathrm{TeV}$.

In the sense of AdS/CFT correspondence [2], this RS1 model is dual 3] to a $4 D$ CFT of which the Higgs is a composite which arises after conformal invariance is broken, i.e., RS1 is dual to a strongly interacting Higgs sector model. Thus, the hierarchy problem is solved by compositeness of Higgs. In addition, the SM gauge and fermions fields are also composites so that flavor issues (and corrections to electroweak precision observables) depend on details of this compositeness (dual to UV sensitivity on RS1 side). In general, we expect FCNC's suppressed by compositeness scale of $\sim \mathrm{TeV}$ without any small coefficients and thus too large.

An alternative to the above is that only Higgs is a composite of a strongly interacting sector 4, in this case a CFT, while the SM gauge and fermions fields are fundamental fields, external to the CFT. This suffices to solve the

hierarchy problem since only the Higgs mass requires protection, the masses of gauge and fermion fields being protected by symmetries. However, for gauge boson and fermion masses to arise at the weak scale, these fields must couple to the CFT/Higgs sector. The RS dual of this $4 D$ set-up is SM gauge and fermion fields in the bulk.

The above UV sensitivity is removed when the fermions and the gauge bosons are allowed to propagate in the bulk. One then uses the idea of split fermions by localizing the light quarks near the Planck brane. This modification of the model yields three important virtues:

(i) Automatic suppression of the higher dimensional operators due to a high 
effective cut-off near Planck brane [5, 6].

(ii) The flavor puzzle is ameliorated, since the quark masses and mixing are determined by the value of their wave function (WF) on the TeV brane 7. [5, 6].

(iii) Since the SM fields are in the bulk presence of new KK states is implied. The coupling of these new states are both custodial and flavor symmetry violating. It turns out however (as we explain below) that flavor dependence in the couplings between these new heavy states and the light fermion modes are suppressed (unlike as in the flat extra dimension case) by combination of RS-GIM mechanism and approximate symmetries.

Constraints from EWPM in the above setup were shown to imply a bound on the KK masses $m_{\mathrm{KK}} \gtrsim \mathcal{O}(10) \mathrm{TeV}[8]$. This bound, combined with virtue (iii), makes the model consistent with constraints from FCNC processes [6]. In spite of this success the model now raises the little hierarchy problem: Fine-tuning is required to explain the smallness of the EWSB scale, $\Lambda_{\mathrm{EWSB}}$, compared to the lightest non-zero KK mass,

$$
\left(\frac{\Lambda_{\mathrm{EWSB}}}{m_{\mathrm{KK}}}\right)^{2}=\mathcal{O}\left(10^{-4}\right) .
$$

Reference [9] (for models with a Higgs) and [10] (for Higgsless models) improved upon the above by promoting the gauge symmetry in the bulk to be custodial invariant. It was shown in 9] that a model with $\mathrm{KK}$ masses of $\mathcal{O}(3 \mathrm{TeV})$ can be consistent with EWPM for the Higgs case. The consistency with EWPM for the Higgsless models was considered in [11, 12, 13, 14, 15, 16] with partial success. In any case, one may expect that the little hierarchy problem would be reduced to the $\mathrm{O}(1 \%)$ bearable range in model with Higgs on $\mathrm{TeV}$ brane and to the $\mathrm{O}(10 \%)$ range in models with Higgs in the bulk (but with a wavefunction peaked near TeV brane) [17]; whereas, there is no fine-tuning in Higgsless models.

\subsection{Overview}

As discussed above the way the zero mode profiles are located in the extra dimension plays an essential role in the success of the above models. This implies that up to some limited freedom the flavor parameters of the framework are fixed. Thus our aim below is to make a systematic study of the structure of flavor violation in this framework. A discussion of the signals for B-physics already appeared in our previous work [18.

We perform our analysis under the assumption that the model contains the minimal amount of fine tuning and hierarchies in its fundamental parameters. In particular we assume low KK masses,

$$
m_{\mathrm{KK}} \lesssim 3 \mathrm{TeV},
$$


and also that the entries in the 5D Yukawa matrices are complex and of the same order. Since the KK masses are smaller than in earlier studies, we expect the FCNC's to be enhanced leading to non-trivial constraints and signals. An earlier study of flavor violation with few TeV KK masses appears in reference 19. However, as we will discuss later, hierarchies in 5D Yukawa were allowed in that study leading to quite different conclusions/signals than in our study.

Having made these assumptions, we then look for signals which will be able to test the model's predictions related to the flavor sector. As it turns out, the structure of flavor violation in the KK theory mostly depends on value of zero mode profiles near the $\mathrm{TeV}$ brane. These are expected to be similar in all the existing models. Consequently our results are robust and independent of details within this class of models.

As mentioned above, there is a combination of RS-GIM mechanism and approximate symmetries for light fermions leading to suppressed FCNC's. We show below that, just as in the SM, the RS-GIM mechanism is violated by large top quark mass. This result in the following three types of new physics (NP) contributions:

(I) Contributions to $\Delta F=2 \mathrm{FCNC}$ processes - arise from a relative large dispersion in the doublets 5D-masses, specifically large coupling of $(t, b)_{L}$ to gauge KK modes due to heaviness of the top quark

(II) Contributions to $\Delta F=1 \mathrm{FCNC}$ processes (mostly semileptonic) - arise from combination of (I) and mixing between the zero and KK states of the $Z$ due to EWSB.

(III) Contributions to radiative B decay (dipole operators) - arise from large $5 D$ Yukawa required to obtain top quark mass combined with large mixing in the right handed down type diagonalization matrix, $D_{R}$.

In addition we also discuss the NP contributions to electric dipole moments $(\mathrm{EDM}) \mathrm{s}$. We find that 1-loop contributions are predicted to be of $O(10)$ larger than the current experimental sensitivity for $m_{K K} \lesssim 4 \mathrm{TeV}$ which implies an RS CP problem.

In section 2 we briefly introduce the framework and describe the models we consider. Section 3] discusses the flavor structure of the framework and presents a spurion analysis for the KK theory. This makes the structure of flavor violation in the theory more transparent and easy to handle. In section 4 we give an interpretation of the flavor structure in the dual CFT. In section 5 we consider the predictions of our framework for various observables related to the above underlying flavor structure.

In particular, in subsection 5.1 we show that the models predict sizable $\Delta F=$ 2 NP contributions which leads to what we denote as the flavor "coincidence" problem. In subsection 5.2 we discuss $\Delta F=1$ contributions and argue the the NP contribution are likely to be observed only in semi-leptonic decays (e. g. $B \rightarrow X_{s} l^{+} l^{-}$). In subsection 5.3 we estimate the NP contributions to various radiative $\mathrm{B}$ decays. In subsection 5.4 we consider the NP contributions to EDMs 
which are related to flavor diagonal CPV. In subsection 5.5 we briefly comment about flavor violation in the up type sector. Finally we conclude in section [6. Appendices contain technical details.

\section{The framework}

We begin with a description of the model independent features of the framework under study $9,10,20,21$. We shall then briefly comment about the differences between the relevant models considered below. The basic set-up of our models is the RS1 framework 1. The space time of the model is described by a slice of $\mathrm{ADS}_{5}$ with curvature scale, $k \sim M_{P l}$, the $4 D$ Planck mass. The Planck brane is located at $\theta=0$, where $\theta$ is the compact extra dimension coordinate. The $\mathrm{TeV}$ brane is located at $\theta=\pi$. The metric of RS1 can be written as:

$$
(d s)^{2}=\frac{1}{(k z)^{2}}\left[\eta_{\mu \nu} d x^{\mu} d x^{\nu}-(d z)^{2}\right]
$$

where $k z=e^{k r_{c} \theta}$. We assume that $k \pi r_{c} \sim \log \left(M_{\mathrm{Pl}} / \mathrm{TeV}\right)$ to solve the hierarchy problem,

$$
\left(z_{h} \equiv \frac{1}{k}\right) \leq z \leq\left(z_{v} \equiv \frac{e^{k \pi r_{c}}}{k}\right)
$$

where $z_{v} \sim \mathrm{TeV}^{-1}$.

The gauge group of the models under study is given by [9, 10] $S U(3)_{c} \times$ $S U(2)_{L} \times S U(2)_{R} \times U(1)_{B-L}$. The gauge symmetry is broken on the Planck brane down to the SM gauge group and in the TeV brane it is broken down to $S U(3)_{c} \times S U(2)_{D} \times U(1)_{B-L} . S U(2)_{D}$ is the diagonal subgroup of the two $S U(2)$ 's present in the bulk.

The fermion and the scalar fields content is model dependent. We shall elaborate more on the fermions sector in the following section. The major role played by the Higgs field, relevant to our consideration, is to yield the masses and mixing for the SM fields and in addition mixing between these and the higher KK fields. In that sense it is not important whether we consider the Higgs 9] or Higgsless models [10, 20, 21]. Thus we will not elaborate more on this subject. In points, however, in which the difference between the models is relevant we shall explicitly specify that.

The Lagrangian of the models can be described by

$$
S=\int d^{4} x d z \sqrt{G}\left[\mathcal{L}_{\text {gauge }}+\mathcal{L}_{\text {fermion }}+\mathcal{L}_{\mathrm{Pl}} \delta\left(z-z_{h}\right)+\mathcal{L}_{\mathrm{TeV}} \delta\left(z-z_{v}\right)\right]
$$

where $\mathcal{L}_{\text {gauge }}+\mathcal{L}_{\text {fermion }}$ is the bulk Lagrangian. The bulk gauge Lagrangian is

$$
\mathcal{L}_{\text {gauge }}=\sqrt{G}\left(-\frac{1}{4\left(g_{5 D}^{i}\right)^{2}} F_{i M N} F_{i}^{M N}\right)
$$


where $g_{5 D}$ is the $5 D$ gauge coupling and the index $i$ runs over the $S U(3)_{c}$, $S U(2)_{L}, S U(2)_{R}$ and $U(1)_{B-L}$ gauge fields. The fermion Lagrangian will be presented in the next section.

The TeV brane Lagrangian contains the EWSB sector (including SM fermion mass terms: see Eq. (6)). The Planck brane Lagrangian, $\mathcal{L}_{P l}$ contains necessary fields to spontaneously break bulk gauge symmetry to $S U(2)_{L} \times U(1)_{Y}$ and also to break degeneracy between up and down quark masses in some models (see below).

\section{$2.1 \quad$ Fermions}

The fermion sector of $\left[9,20\right.$ is described as: $Q=(3,2,1)_{\frac{1}{3}}, u=(3,1,2)_{\frac{1}{3}}, d=$ $(3,1,2)_{\frac{1}{3}}$, where the number in the parenthesis stands for the fermion representation under the $S U(3)_{c} \times S U(2)_{L} \times S U(2)_{R} \times U(1)_{B-L}$ gauge group respectively, and all of them propagate in the bulk. We use the notation $Q, u, d$ to match with the transformation of the light modes, $Q^{0}, u^{0}, d^{0}$, belonging to the above fields under the SM gauge group. ${ }^{4}$ Thus $Q$ contains two SM-light fields and $u, d$ contain each a single SM-light field. The fermion sector of [21] consist of $Q=(3,2,1)_{\frac{1}{3}}, Q_{R}=(3,1,2)_{\frac{1}{3}}$, where in that case $Q_{R}$ contains both the SM up and down type singlets. In addition, to break the degeneracy between the two light components of $Q_{R}$ a pair of Planck brane vector like quarks was added. These couple to the up type singlet component of $Q_{R}$. Consequently its WF is distorted and splitting between the up and down SM singlet quarks is achieved.

At the end of the day, one finds that to account for the SM masses and mixing the profile of the zero modes in 21] is similar to the ones in 9, 20]. This is one of the main reasons for the fact that our analysis is robust and model independent. In order to do actual calculation we arbitrarily choose to work with the fermion sector of [9, 20]. We nevertheless have in mind that our analysis can be straightforwardly translated into the language of [21.

\section{Flavor Structure}

\subsection{Flavor violation - 5D theory}

The relevant piece of the Lagrangian, related to the flavor sector, is given by a bulk piece

$$
\mathcal{L}_{\text {fermion }}=\sqrt{G}\left[i \bar{\Psi}_{i} \Gamma^{M} D_{M} \Psi_{i}+k C_{Q, u, d}(\bar{Q}, \bar{u}, \bar{d})(Q, u, d)\right]
$$

(the index $i$ runs over the different fermion representations) and a TeV-brane localized piece:

$$
\mathcal{L}_{T e V} \ni h \delta\left(z-z_{v}\right) \lambda_{u, d 5 D} \bar{Q}(u, d),
$$

where for the Higgs models $h$ stands for the Higgs field and for the Higgsless ones it stands for a mass parameter $h \rightarrow\langle h\rangle=v \simeq 174 \mathrm{GeV}$. In addition $C_{Q, u, d}$

\footnotetext{
${ }^{4}$ We use the terms zero modes and light modes for the SM fields interchangeably.
} 
are $3 \times 3$ hermitian matrices (due to $5 \mathrm{D}$ Lorentz symmetry) and $\lambda_{u, d 5 D}$ are the $5 \mathrm{D}$ Yukawa matrices. In principle, since the above theory is non-renormalizable, higher dimension, flavor violating, operators should be present in (6). Due to the fact that the light quarks are localized near the Planck brane the effective cut-off relevant to this type of operators is very high. Thus they are subdominant and are neglected in our analysis below.

Unlike the SM case, in addition to the Yukawa matrices the model contains also additional sources of flavor violation in the form of $C_{Q, u, d}$.

$$
\mathrm{U}(3)_{Q, u, d} \stackrel{C_{Q, u, d}}{\longrightarrow} \mathrm{U}(1)^{3}{ }_{Q, u, d},
$$

where $\mathrm{U}(3)_{Q, u, d}$ is the flavor group of the $5 \mathrm{D}$ theory, per representation, in the limit where $C_{Q, u, d}, \lambda_{u, d 5 D} \rightarrow 0$. Indeed as discussed below the addition of these matrices induces the non-trivial flavor structure of this framework.

One can count the number of the physical flavor parameters present in the above theory. Generically, however, there is no direct translation between the flavor parameters of the 5D theory and the ones appearing in the IR limit of the theory. This is the case since it depends on integrals of $z$ dependent functions. Thus the quantities which characterize the flavor violation in the $4 \mathrm{D}$ effective theory are functionals of $C_{Q, u, d} \cdot{ }^{5}$ In the RS framework, as discussed below, there is, in fact, a simple relation between the structure of flavor violation in the $5 \mathrm{D}$ and $4 \mathrm{D}$ theories.

Thus it is worthwhile to do the above counting. For reasons that will be clear below it is useful to separate the counting into the following two cases. We start with the case in which flavor violations occur only in the down type quark sector, $C_{u}, \lambda_{u 5 D} \rightarrow 0$. Then we consider the generic case. ${ }^{6}$

(i) Down type quark sector - $\lambda_{d 5 D}$ contains 9 real and 9 imaginary parameters and $C_{Q, d}$ contains, each, 6 real and 3 imaginary parameters. Altogether we find 21 real and 15 imaginary parameters. The $U(3)_{Q, d}$ global symmetries can be use to eliminate, however, 6 real and 11 phases which are unphysical (one phase cannot be removed since it corresponds to an unbroken baryon number symmetry.). Thus after applying the above rotation of the fields we find 15 real and 4 imaginary physical flavor parameters. This case is summarized in table 1 .

(ii) Generic model $-\lambda_{u, d 5 D}$ contains 18 real and 18 imaginary parameters and $C_{Q, u, d}$ contains 18 real and 9 imaginary parameters. Altogether we find 36 real and 27 imaginary parameters. The $U(3)_{Q, u, d}$ symmetries can be used to eliminate 9 real and 17 phases. Thus, we find 27 real and 10 imaginary physical flavor parameters. This case is summarized in table 2

The physical role of these parameters is obscure at this level. We shall see, however, that in the KK theory the role of the above parameters is more transparent.

\footnotetext{
${ }^{5}$ This is generically the situation in any $5 \mathrm{D}$ theory which realizes the split fermions idea.

${ }^{6}$ For more details on counting flavor parameters see e.g. 22 and refs therein.
} 


\section{$3.2 \quad$ Flavor violation - KK theory}

\subsubsection{Zero modes and SM flavor parameters}

The fermion zero-modes are identified with the observed SM fermions. As explained in the introduction our working assumption is that the 5D Yukawa matrices are anarchical. The hierarchy in SM flavor parameters is, therefore, directly related to the zero mode profiles in the $5 \mathrm{D}$ through the split fermion mechanism. In order to see how this works we start with considering only the zero modes sector of our framework.

We can always do actual computations in a basis in which the bulk masses are diagonal, $C_{Q, u, d}=\operatorname{diag}\left(c_{Q^{i}, u^{i}, d^{i}}\right)$. In this basis, we shall denote as the "special basis" the WF of the, canonically normalized, zero-mode fermions are given by (see, for example, [].

$$
\hat{\chi}_{0}\left(c_{x^{i}}, z\right) \equiv z^{-\frac{3}{2}} \chi_{0}\left(c_{x^{i}}, z\right)=\sqrt{\frac{k\left(1-2 c_{x^{i}}\right)}{\left(k z_{v}\right)^{1-2 c_{x^{i}}}-1}}(k z)^{\frac{1}{2}-c_{x^{i}}},
$$

where $\chi_{0}\left(c_{x^{i}}, z\right)$ is the zero mode profile, $x=Q, u, d$, and in the above we neglected electroweak breaking effects on the TeV brane. It is evident from (7) that when $c_{x^{i}}>1 / 2\left(c_{x^{i}}<1 / 2\right)$ the zero-mode fermion is localized near Planck $(\mathrm{TeV})$ brane.

Using (66) we find that the effective $4 \mathrm{D}$ Yukawa matrices $\lambda_{u, d 4 D}$ are given by:

$$
\lambda_{u, d 4 D}^{i j}=\frac{2 \lambda_{u, d 5 D}^{i j} k}{f_{Q^{i}} f_{u^{j}, d^{j}}},
$$

where

$$
\frac{2 k}{f_{x^{i}}^{2}}=\hat{\chi}_{0}^{2}\left(c_{x^{i}}, z_{v}\right)
$$

It is useful to find the asymptotic dependence of $f_{x^{i}}$ on $c_{x^{i}}$

$$
f_{x^{i}}^{-2} \sim \begin{cases}\frac{1}{2}-c_{x^{i}} & \text { for } c_{x^{i}}<\frac{1}{2}-\epsilon \\ \frac{1}{2 k \pi r_{c}} & \text { for } c_{x^{i}} \rightarrow \frac{1}{2} \\ \left(c_{x^{i}}-\frac{1}{2}\right) e^{k \pi r_{c}\left(1-2 c_{x^{i}}\right)} & \text { for } c_{x^{i}}>\frac{1}{2}+\epsilon,\end{cases}
$$

\begin{tabular}{||c|c|c||}
\hline \hline & $\operatorname{Re}$ & $\mathrm{Im}$ \\
\hline$\lambda_{d 5 D}$ & 9 & 9 \\
\hline$c_{Q}+c_{d}$ & 12 & 6 \\
\hline Total & 21 & 15 \\
\hline Non-phys' parameters & 6 & 11 \\
\hline Phys' parameters & 15 & 4 \\
\hline \hline
\end{tabular}

Table 1: Number of flavor parameters for a theory with only down type quark sector. 


\begin{tabular}{||c|c|c||}
\hline \hline & $\mathrm{Re}$ & $\mathrm{Im}$ \\
\hline$\lambda_{u 5 D}+\lambda_{d 5 D}$ & $2 \times 9$ & $2 \times 9$ \\
\hline$c_{Q}+c_{u}+c_{d}$ & $3 \times 6$ & $3 \times 3$ \\
\hline Total & 36 & 27 \\
\hline Non-phys' parameters & 9 & 17 \\
\hline Total phys' parameters & 27 & 10 \\
\hline \hline
\end{tabular}

Table 2: Total number of flavor parameters in the full theory.

where $\epsilon \sim 0.1$ so that the asymptotic value of $f_{x^{i}}$ is obtained rapidly for $c>(<$ ) $1 / 2$.

Generically $\lambda_{u, d 5 D}$ are expected to be anarchical. We therefore assume that all the entries in these Yukawa matrices are complex and of order unity. As a consequence the hierarchy in the SM flavor parameters should be accounted for by the corresponding values of $f_{Q, u, d}$. Up to an overall dimensionful proportionality coefficient, $\lambda_{5 D}$, the following relation between $f_{Q, u, d}$, and the flavor parameters should hold,

$$
m_{u^{i}, d^{i}} \sim \frac{2 v \lambda_{5 D} k}{f_{Q^{i}} f_{u^{i}, d^{i}}},
$$

where $v \simeq 174 \mathrm{GeV}$. Furthermore, the size of the elements of $U_{L, R},\left(D_{R, L}\right)$ the diagonalization matrices of the up (down) Yukawa matrices (related to the SM doublet and singlets field respectively) are given by:

$$
\begin{aligned}
& \left|\left(D_{L}\right)_{i j}\right| \sim\left|\left(U_{L}\right)_{i j}\right| \sim\left|\left(V_{\mathrm{CKM}}\right)_{i j}\right| \sim \frac{f_{Q^{i}}}{f_{Q^{j}}} \quad \text { for } \quad j \leq i, \\
& \left|\left(U_{R}, D_{R}\right)_{i j}\right| \sim \frac{f_{u^{i}, d^{i}}}{f_{u^{j}, d^{j}}} \quad \text { for } \quad j \leq i,
\end{aligned}
$$

where $V_{\mathrm{CKM}} \equiv U_{L}^{\dagger} D_{L}$, and in the above for $j>i$ one should interchange the $i$ and $j$ indices.

\subsubsection{Fixing the values of $f_{Q, u, d}$}

Eqs. (1112) provide eight relations, between the flavor observables, for the nine flavor parameters, $f_{x^{i}}$, and the overall scale $\lambda_{5 D}$. In order to maintain the perturbativity of the theory $f_{x^{i}}\left(\lambda_{5 D}\right)$ cannot be arbitrarily small (large) as follows. For the theory to contain at least two KK modes before it becomes strongly coupled we require [9] ${ }^{7}$

$$
\lambda_{5 D} k \lesssim 4
$$

\footnotetext{
${ }^{7}$ In 13 higher value of $\lambda_{5 D}$ are considered. This implies that the the KK modes are strongly coupled. Since we will consider this kind of coupling in our analysis below, we cannot trust our results in that range. Following the spirit of [13, however, who argue that in the above limit nothing special is expected to happen to observable quantities we claim that our conclusions should likewise hold in the above range.
} 
$f_{x^{i}}$ cannot be smaller than unity since it implies that the corresponding bulk mass, $k C_{x^{i}}$, exceeds the curvature scale so that $\Psi_{x^{i}}$ should be treated as a brane localized fermion. In addition to have a sufficiently heavy partner for the $S U(2)_{R}$ partner of $t_{R}$ (in order to avoid large shift in coupling of $b_{L}$ to $Z$ via its mass mixing with $b_{L}$ ) requires 9

$$
f_{u^{3}} \gtrsim 1.2 .
$$

Note that constraint from EWPM $(Z \rightarrow b \bar{b})$ typically requires [9, 13] $f_{Q^{3}} \gtrsim 2$ regardless of flavor mixing. We will be careful in what follows to correlate (incorporate) this constraint with (into) our flavor analysis. In the previous study with few TeV KK masses [19], such correlation was not studied. Thus, by itself, this bound combined with the known value of $m_{t}$ and the lower bound of (14) effectively yield a lower bound on $\lambda_{5 D}$, of $\mathcal{O}\left(\frac{3}{k}\right)$. This is close to upper bound of (13), which is important for our results related to radiative $\mathrm{B}$ decays (see subsection 5.31). As for constraint from FCNC processes we shall see below that the smaller $f_{Q^{3}}$ is the larger are the NP contributions.

Consequently, to derive lower bound on these contributions we shall use the lower bound of (14) and upper bound of (13) which gives maximum value of $f_{Q^{3}}$ of $\mathcal{O}(3)$.

Having fixed the values of $\lambda_{5 D}$ and $f_{u^{3}}$ we can use eqs. (11) and (12) to solve for the other eight parameters. In table 3 we summarize the related relation between these parameters and the resultant values. At this point, neglecting effects of higher KK fields, we have all the information regarding the IR limit of the theory. This is however not very interesting since apart from the scalar sector, which is model dependent, it is equivalent to the SM. In order to study its deviation from the SM we need to consider the higher KK modes.

\begin{tabular}{||c|l|l|l||}
\hline \hline Flavor & $f_{Q}^{-1}$ & $f_{u}^{-1}$ & $f_{d}^{-1}$ \\
\hline \hline I & $\frac{\lambda^{3}}{f_{Q^{3}}} \sim 0.4 \times 10^{-2}$ & $\frac{m_{u}}{m_{t}} \frac{f^{-1}}{\lambda^{3}} \sim 10^{-3}$ & $\frac{m_{d}}{m_{b}} \frac{f_{d^{3}}^{-1}}{\lambda^{3}} \sim 10^{-3}$ \\
\hline II & $\frac{\lambda^{2}}{f_{Q^{3}}} \sim 2 \times 10^{-2}$ & $\frac{m_{c}}{m_{t}} \frac{f_{u^{3}}^{-1}}{\lambda^{2}} \sim 10^{-1}$ & $\frac{m_{s}}{m_{b}} \frac{f_{d^{3}}^{-1}}{\lambda^{2}} \sim 0.3 \times 10^{-2}$ \\
\hline III & $\frac{f_{u}^{3} m_{t}}{\lambda_{5} k} \sim \frac{1}{3}$ & $\mathbf{O}\left(\frac{5}{6}\right)$ & $\frac{m_{b}}{m_{t}} f_{u^{3}}^{-1} \sim 0.6 \times 10^{-2}$ \\
\hline \hline
\end{tabular}

Table 3: The known quark masses and CKM mixing implies relation between the model flavor parameters, $f_{x^{i}}$, (1112). The value of $f_{u^{3}}, \lambda_{5 D}$ is determined by requiring the theory is perturbative (1314).

\subsubsection{Flavor violation with KK modes}

In generic split fermions models the flavor structure of the KK theory is complicated and cannot be calculated analytically. The bulk RS1 framework, however has several unique feature which makes, at leading order, the flavor structure of the KK theory extremely simple. The above structure is understood from 
the above observation: At leading order, all the profiles of the $K K$ modes are localized near the TeV brane.

This implies that flavor violating coupling are "KK blind". In particular, $c$ 's for all SM fermions (except for $t_{R}$ which plays a minor role in low energy flavor dynamics) are close to $1 / 2$ so that one finds the following features for the fermion KK excitations:

(i) Up to small corrections (due to difference in widths), for a given KK level, all the KK excited fermion profiles are the same.

(ii) The masses of the three generations for each KK level, are degenerate, up to a small correction.

This holds to a very good approximation as shown in Appendix $\mathbb{B}$ for the fermion ones. The reason behind this result is that KK spectrum and wavefunctions are not sensitive to this (minor) variation in $c$ (in contrast to the zero-mode wavefunction, which is very sensitive to $c$ ).

Our next step is to explicitly present the part of the KK Lagrangian yielded after we apply the integral over $z$. This part describes the flavor structure of the theory including interactions with the higher KK modes.

$$
\mathcal{L}_{\mathrm{KK}}=\mathcal{L}_{\mathrm{KK}}^{g}+\mathcal{L}_{\mathrm{KK}}^{Y}+\mathcal{L}^{Z},
$$

where $\mathcal{L}_{\mathrm{KK}}^{g}$ contains the interaction with the higher KK gauge bosons, $\mathcal{L}_{\mathrm{KK}}^{Y}$ contains interactions with higher KK fermions and $\mathcal{L}^{Z}$ contains the flavor violating part due to EWSB effects. After integrating over $z$ the gauge interactions part, in the "special basis", is of the form

$$
\begin{aligned}
\mathcal{L}_{\mathrm{KK}}^{g} \sim \sum_{x, i} \sqrt{k \pi r_{c}} & g_{x} \sum_{n} G^{n}\left[\psi_{x^{i}}^{0 \dagger} \psi_{x^{i}}^{0}\left(\frac{1}{k \pi r_{c}}+\frac{1}{f_{x^{i}}^{2}}\right)\right. \\
& \left.+\sum_{m}\left(\frac{1}{f_{x^{i}}} \psi_{x^{i}}^{0 \dagger} \psi_{x^{i}}^{m}+\sum_{p} \psi_{x^{i}}^{m \dagger} \psi_{x^{i}}^{p}+h . c\right)\right]
\end{aligned}
$$

where $\psi_{Q, u, d}^{l}$ is a $4 \mathrm{D}$ fermion field, $i$ is a flavor index, $l, m, n$ stands for the corresponding KK levels, $g_{x}$ stands for the three $4 D / \mathrm{SM}$ gauge couplings (see Eq. (60),$G^{n}$ is a KK gauge boson and we suppressed the Lorenz structure. In addition, we neglected EWSB effects which are separately discussed below.

The Yukawa part is of the form ${ }^{8}$

$\left.\mathcal{L}_{\mathrm{KK}}^{Y} \approx h \sum_{m, i, j} 2 \lambda_{u, d 5 D k}^{i j}\left(\frac{\psi_{Q^{i}}^{0 \dagger}}{f_{Q^{i}}} \psi_{u^{j}, d^{j}}^{m}+\psi_{Q^{i}}^{m \dagger} \frac{\psi_{u^{j}, d^{j}}^{0}}{f_{u^{j}, d^{j}}}+\sum_{n} \psi_{Q^{i}}^{m \dagger} \psi_{u^{j}, d^{j}}^{n}+h . c\right)\right|_{\mathrm{TeV}}$.

\footnotetext{
${ }^{8}$ We shall work in the mass insertion approximation. That is we shall treat the Yukawa interactions/mass terms on the $\mathrm{TeV}$ brane as a perturbation.
} 


\subsubsection{Flavor violation in $Z$ coupling from EWSB}

In our framework, with or without the Higgs, EWSB occurs only at the boundaries of the extra dimension. This leads to an important effect relevant to our considerations. That is, it distorts profile of the physical $Z$ near the TeV brane $8,0,13$. Its profile is given by a linear combination of the undistorted KK states; where the mixing angle, $\delta g_{Z}$, between the ordinary basis and the distorted one is of order of

$$
\delta g_{Z} \sim \sqrt{k \pi r_{c}}\left(\frac{M_{Z}}{m_{\mathrm{KK}}}\right)^{2}=\mathcal{O}(1 \%) .
$$

Below we shall only be interested in the flavor violating part of the $Z$ coupling to two SM fermions. Thus the relevant part of the Lagrangian for this case is given by (16)

$$
\mathcal{L}^{Z} \sim \sum_{x, i} \sqrt{k \pi r_{c}} \delta g_{Z} \frac{g_{2}}{2 \cos \theta_{\mathrm{W}}} Z \psi_{x^{i}}^{0 \dagger}\left(v_{f}-\gamma_{5} a_{f}\right) \psi_{x^{i}}^{0} \frac{1}{f_{x^{i}}^{2}},
$$

where $a_{f}=T_{3}^{f}$ and $v_{f}=T_{3}^{f}-Q_{3}^{f} \sin ^{2} \theta_{\mathrm{W}}$.

\subsection{Flavor violation and spurion analysis}

Using the values of the flavor parameters in table 3 and the flavor structure of the theory given in eqs. (16 1718) the model is fully determined. At this point, in principle, one can derive a prediction for any process which is related to the flavor sector of the theory. It is very instructive, however, to note that the above framework has an underlying organized structure. It is linked with our above observation that, to leading order, flavor violation appear in a universal way in the KK couplings.

We can summarize the relation in eqs. (16/17/18) by the following:

- Flavor violation in coupling between KK modes stems only from the Yukawa matrices $\lambda_{u, d 5 D}$.

- Flavor violation between a zero mode and other fields is always accompanied by a factor of $f_{x^{i}}^{-1}$.

So far all our analysis [in particular (161718)] was done in the "special basis" in which the $5 \mathrm{D}$ bulk masses are diagonal. In order to get more insight into the pattern of the flavor violating interactions we consider the global symmetries of the above KK theory in various limits.

Switching off all the interactions we find the following large flavor symmetry

$$
\mathrm{U}(3)_{Q, u, d} \times \Pi_{n} \mathrm{U}(3)_{Q, u, d}^{n},
$$

where the first term stands for the SM flavor group and the second stands for product of groups, one for each KK level (per representation). Omitting, for a 
moment, the zero modes we find that the 5D Yukawa matrices break the above symmetries as follows

$$
\mathrm{U}(3)_{Q}^{n} \times \mathrm{U}(3)_{u, d}^{m} \stackrel{\lambda_{u, d 5 D}}{\longrightarrow} \mathrm{U}(1)^{3}{ }_{u^{\prime}, d^{\prime}}, \quad n, m \neq 0 .
$$

This implies that we can think of the 5D Yukawa matrices as, spurion fields, bi-fundamentals of the diagonal KK flavor group, $\mathrm{U}(3)_{Q}^{n} \times \mathrm{U}(3)_{u, d}^{n}$,

$$
\lambda_{u, d 5 D}=\left(\overline{\mathbf{3}}_{Q}^{n}, \mathbf{3}_{u, d}^{n}\right) .
$$

As discussed above, the only way zero modes can couple to other fields (we are not interested in the flavor universal pieces) is through extra factors of $f_{x^{i}}^{-1}$. Thus from eqs. (161718) we find the following breaking pattern

$$
\mathrm{U}(3)_{Q, u, d} \times \mathrm{U}(3)_{Q, u, d}^{n} \stackrel{f_{Q^{i}, u^{i}, d^{i}}^{-1}}{\longrightarrow} \mathrm{U}^{\mathrm{D}}(1)^{3}{ }_{Q, u, d},
$$

where $\mathrm{U}(3)_{Q, u, d}^{n}$ stands again for the diagonal $\mathrm{KK}$ flavor group and $\mathrm{U}^{\mathrm{D}}(1)$ is in the diagonal basis of the KK and SM flavor groups. This implies that we can view the $f_{x^{i}}^{-1}$ as eigenvalues of a matrix, we denote as $F_{x}$, where in the "special basis" we have

$$
F_{x} \equiv \operatorname{diag}\left(f_{x^{i}}^{-1}\right)
$$

We learn then that $F_{x}$ transforms as a bi-fundamental under the $\mathrm{U}(3)_{Q, u, d} \times \mathrm{U}(3)_{Q, u, d}^{n}$ flavor group

$$
F_{Q}^{\dagger}, F_{u, d}=\left(\overline{\mathbf{3}}_{Q, u, d}^{n}, \mathbf{3}_{Q, u, d}\right) .
$$

We finish this part by noting that there are two interesting limits regarding the spurions $F_{Q, u, d}$.

(i) $F_{x^{i}} \rightarrow 0$ - the SM flavor group is unbroken. Looking at the values of $f_{x^{i}}^{-1}$ given in table 3 we find the following feature. All the values of the $f_{x^{i}}^{-1}$ apart from the ones related to the top mass are small. This implies that the model has a built-in approximate flavor symmetry for the light quarks. This is indeed the reason why the framework may avoid the severe constraint from FCNC processes with such a low KK masses. We can compare this with the flat extra dimension models which require $\mathrm{KK}$ masses of $\mathcal{O}(1000 \mathrm{TeV})$.

(ii) $F_{x} \rightarrow$ constant $\times \mathbf{1}_{3}$ - the $\mathrm{U}(3)_{Q, u, d} \times \mathrm{U}(3)_{Q, u, d}^{n}$ flavor group is broken to a $U(3)$ diagonal one. This means that to have flavor violation (not through SM Yukawa interactions) a non-degeneracy in $F_{Q, u, d}$ is required. This is the RS-GIM mechanism and as discussed above only top related entries in $F_{Q, u}$ induce sizable RS-GIM violation. RS-GIM mechanism is violated by 1 st and 2 nd generation as well in our framework since $F_{Q, d}$, 's are nondegenerate. However, $F_{Q}, d$, $u$ 's are small so that violation of RS-GIM is not severe, i.e., FCNC's are protected by built-in approximate symmetries. Also, the 2nd limit corresponds to minimal flavor violation (MFV) since only source of FV is $5 D$ Yukawa which is the same spurion as the $4 D$ Yukawa since $F \propto \mathbf{1}_{3}$. 


\subsubsection{Relations among couplings and non-trivial predictions}

In the KK theory the number of vertices with nontrivial flavor structure is large. According to our approximation that flavor violation is KK blind, these interactions are described by only five spurions $F_{Q, u, d}$ and $\lambda_{u, d 5 D}$. Consequently these couplings are not independent and there are relations among them. One such trivial relation is that the Yukawa interactions between any two KK fermions, with KK levels

$n, m$, (and the Higgs), $\lambda_{u, d}^{n m}$, are just proportional to a single spurion $\lambda_{u, d 5 D}$,

$$
\lambda_{u, d}^{n m} \propto \lambda_{u, d 5 D} .
$$

The ones that are relevant to our work contain at least a single zero mode leg. These have less trivial relations among them. For example the product of a gauge interaction between a zero mode and an $\mathrm{n}$ KK fermion (and a KK gluon) and a Yukawa coupling between $\mathrm{n}$ and $\mathrm{m} \mathrm{KK}$ fermions (and the Higgs) is proportional to the direct Yukawa coupling between a zero mode and an $\mathrm{m}$ level KK fermion (1617):

$$
g_{Q}^{0 n} \lambda_{u, d}^{n m}, \lambda_{u, d}^{n m} g_{u, d}^{m 0} \propto g F_{Q} \lambda_{u, d 5 D}, g \lambda_{u, d 5 D} F_{u, d} \propto \lambda_{u, d}^{0 m}, \lambda_{u, d}^{m 0}
$$

We can use the above to argue that the KK gluon diagram shown in fig. 4. [discussed below in subsection [5.3)] yields a small flavor-violating effect since it is aligned with the down type mass matrix

$$
g_{Q}^{0 m} \lambda_{d 5 D} g_{d}^{n 0} \propto \lambda_{d 4 D} .
$$

This basically explains why the lowest order, sizable, contribution to chirality flipping operators that we find in subsection (5.3) is proportional to $\mathcal{O}\left(\lambda_{u, d 5 D}^{3}\right)$.

We finish this part by pointing out that the above relations yield remarkable correlation between measurements of observables in low energy experiments and ones related to high energy theory specific to this framework. In principle just based on low energy experiments (and top mass measurement) we can determine all the model flavor parameters i.e. the values of $f_{x^{i}}$, the mixing angle and the $\mathrm{CP}$ phases. Consequently, using relations similar to the ones in eqs. (1920), we will (in principle) be able to completely predict the amplitude for high energy processes in which incoming or outgoing, on shell, KK quarks are participating!

\subsubsection{Counting parameters in the KK theory}

The above analysis shows yet another special feature of the RS1 framework. That is we can directly translate the flavor violating parameters in the $5 \mathrm{D}$ theory to the ones appearing in the couplings of the KK theory. In the "special basis" this is transparent since there is one to one correspondence between the eigenvalues of $C_{Q, u, d}$ (and $\lambda_{u, d 5 D}$ ) and $F_{Q, u, d}$ (and $\lambda_{u, d 5 D}$ ) which are the flavor violating sources in the $5 \mathrm{D}$ and the KK theories respectively. Note that in flat extra dimension models there is no such simple correspondence since flavor 
violation in the KK theory is found by calculating overlap-integrals between the WF's of the fields.

Let us verify that statement by counting the flavor parameters in the KK theory and see that we can reproduce our results derived in the 5D theory summarized in tables 1 and 2

As done in subsection 3.1] we start with the case in which flavor violations occur only in the down type quark sector, $F_{u}, \lambda_{u 5 D} \rightarrow 0$. Then we consider the generic case.

(i) Down type quark sector - Flavor violation is encoded in three generic $3 \times 3$ matrices, $F_{Q, d}$ and $\lambda_{d 5 D}$ which contain altogether 27 real and 27 imaginary parameters. We can use the diagonal KK and the SM flavor symmetries, $\mathrm{U}(3)_{Q, d}^{n} \times \mathrm{U}(3)_{Q, d}$, to eliminate $4 \times 6-1=23$ phases [there is still a conserved $\mathrm{U}(1)$ baryon symmetry in the full theory]. Thus altogether we find four physical phases as in table 1 Two of these are CKM like phase in $D_{L, R}$ and the other two are "Majorana-like", flavor diagonal, and can be shifted between $D_{L, R}$, (for more details see appendix C.3). Similarly we can remove $4 \times 3=12$ real parameters out of the 27 ones and hence 15 physical real parameters are left as in table 1. The real parameters are three quark masses, six mixing angles related to $D_{L, R}$ and the six eigenvalues of $F_{Q, d}$ which measure the non-universal couplings between the different generations and the KK gauge fields.

(ii) Generic model - Flavor violation is encoded in five generic $3 \times 3$ matrices, $F_{Q, u, d}$ and $\lambda_{u, d 5 D}$ which contain 45 real and 45 imaginary parameters. We can use the diagonal KK and the SM flavor symmetries, $\mathrm{U}(3)_{Q, u, d}^{n} \times$ $\mathrm{U}(3)_{Q, u, d}$, to eliminate $6 \times 6-1=35$ phases [there is still a conserved $\mathrm{U}(1)$ baryon symmetry in the full theory]. Thus we find ten physical phases as in table 2 Four of these are CKM like phases in $U_{L, R}, D_{L, R}$ and the other six are "Majorana-like" and can be shifted between $D_{L, R}, U_{L, R}$, (for more details see appendix C.3. ${ }^{9}$ Similarly we can remove $6 \times 3=18$ real parameters out of the 45 ones and hence 27 physical are left as in table 2 The real parameters are six quark masses, twelve mixing angels related to $U_{L, R}, D_{L, R}$ and the nine eigenvalues of $F_{Q, u, d}$.

The last point that we want to make here is related to flavor diagonal CPV. We demonstrate that unlike, for example in generic SUSY models, this framework does not contain flavor diagonal phases in the sense that, without flavor mixing, the Majorana phases mentioned above do not contribute to EDMs, i.e., are not physical. This can be shown by considering the limit in which flavor violation is absent. In that case, all the ten CPV phases can be removed and are not physical: No flavor violation implies that the spurions $\lambda_{u, d 5 D}$ and $F_{Q, u, d}$ (or $\left.C_{Q, u, d}\right)$ can be diagonalized simultaneously. Then one can use a chiral rotation to remove the phases in the Yukawa matrices and to eliminate all the phases from the theory (apart from the strong CP phase).

\footnotetext{
${ }^{9}$ Note that we treat $V_{\mathrm{CKM}}=U_{L}^{\dagger} D_{L}$ and also the analogue matrix which appear in the RH charged currents, $V_{\mathrm{CKM}}^{R}=U_{R}^{\dagger} D_{R}$, as dependent matrices to avoid double-counting of phases.
} 


\section{CFT interpretation of flavor structure of RS1}

In this section, we will show that, remarkably, there is an understanding of flavor structure/built-in approximate symmetries in CFT picture as well. The dual description of this RS1 model has been discussed before (see, for example, references [23, 9, 24] in addition to 3]). For completeness, we will review this description and then describe the flavor structure in CFT picture which has not been discussed in detail before.

As per AdS/CFT correspondence, RS1 is dual to a strongly coupled CFT of which the minimal Higgs is a composite arising after conformal invariance is broken. The SM gauge and fermions fields originate as fundamental fields/external to CFT, but coupled to the CFT/Higgs sector. Due to this coupling, these external fields mix with CFT composites, the resultant massless states correspond to the SM gauge and fermion fields (these are dual to the zero-modes on RS1 side). The degree of this mixing depends on the anomalous/scaling dimension of the CFT operator they couple to. The coupling of SM gauge bosons and fermions to Higgs goes via their composite component since Higgs is a composite of the CFT. Thus, this coupling of fundamental gauge and fermion fields to CFT operators is essential for gauge boson and fermion masses to arise at the weak scale.

\subsection{Duality at qualitative level}

We begin with a qualitative description of dual CFT. The dual interpretation of gauge fields in bulk is that the $4 D$ CFT has a conserved global symmetry current (which is a marginal operator, i.e., zero anomalous dimension) coupled to a $4 D$ gauge field: $A_{\mu} J_{C F T}^{\mu}$. This is just like the photon coupling to a $U(1)_{e m}$ global symmetry current of QCD.

The operator $J_{C F T}^{\mu}$ interpolates/creates out of the vacuum massive spin-1 composites of CFT ("techni- $\rho$ 's" in case of, global, electroweak symmetry of CFT). These composites are similar to $\rho$-mesons in real QCD and are dual to gauge KK modes on RS1 side.

Similarly, the dual interpretation of a bulk fermion is that there is a fundamental fermion (external to CFT) coupled to fermionic CFT operator: $\psi \mathcal{O}_{C F T}$. The operator $\mathcal{O}_{C F T}$ interpolates/creates out of vacuum masssive spin-1/2 composites (just like $J_{C F T}^{\mu}$ creates spin-1 composites) which are dual to fermion KK modes on RS1 side.

The $c$ parameters (bulk fermion masses) are dual to scaling dimension of $\mathcal{O}_{C F T}$ 's which control the mixing of fundamental fermions with CFT composites. The choice of $c>1 / 2$ for light fermions is dual to a coupling $\psi \mathcal{O}_{C F T}$ being irrelevant so that the mixing of $\psi$ with composites is small, i.e., the corresponding SM fermion is mostly fundamental. Thus, the coupling of SM fermion to composite Higgs and also to spin- 1 composites is small since both couplings have to go via the small mixing: the small coupling to $\rho$ 's suppresses FCNC's from their tree-level exchange ${ }^{10}[9$. This agrees qualitatively with small $4 D$ Yukawa

${ }^{10}$ This is the flavor-dependent part of the coupling. There's also a universal coupling induced 
and small flavor-dependence in the coupling to gauge $\mathrm{KK}$ mode obtained on $5 \mathrm{D}$ side. Thus, it is easy to see how the notions of approximates symmetries and RS-GIM arise in the CFT picture.

Similarly one can see the tension arise for the third generation as follows. The SM top quark should have large composite component so that it has $O(1)$ coupling to the composite Higgs, i.e., fundamental top quark should have relevant coupling to CFT (dual to $c<1 / 2$ ). ${ }^{11}$ However, if the fundamental $(t, b)_{L}$ has relevant couplings to the CFT sector, then $\mathrm{SM} b_{L}$ will have large couplings to $\rho$-mesons (due to large mixing of fundamental $b_{L}$ with composites) leading to a shift in coupling of $\mathrm{SM} b_{L}$ to $Z$. So, $Z \rightarrow b \bar{b}$ requires that coupling of fundamental $(t, b)_{L}$ to CFT be at most mildly relevant (dual to $c \sim 0.3-0.4$ ). Nonetheless, the coupling of SM $b_{L}$ to $\rho$-mesons is still larger than that of light fermions and there $i s$ a small shift in coupling of SM $b_{L}$ to $Z$, leading to FCNC's discussed earlier.

Also, to obtain $\lambda_{t} \sim 1$ with only mildly relevant coupling of $t_{L}$ requires that the coupling of the fundamental $t_{R}$ to the CFT sector must be more relevant, which is dual to $c$ for $t_{R} \lesssim 0$. Thus, SM $t_{R}$ contains a sizable admixture of composites.

We see that particles localized near the TeV brane $\left(t_{R}\right.$ zero-mode, Higgs, all KK modes) are (mostly) composites in the CFT picture. This is expected since $\mathrm{TeV}$ brane corresponds to the IR of the CFT. Thus particles which are localized near that brane correspond to IR degrees of freedom in (i.e., composites of) CFT. Similarly, particles localized near Planck brane (light fermion zero-modes) are (mostly) fundamental/external in the CFT picture. This is expected since Planck brane corresponds to the UV of the CFT so that particles localized near that brane correspond to UV degrees of freedom in the CFT picture.

\subsection{Duality at semi-quantitative level}

So far, the CFT description (including the dual understanding of flavor structure/builtin approximate symmetries of the RS model) was qualitative. In this section, we will obtain a semi-quantitative understanding of flavor structure/RS-GIM, in particular, Eqs. (16) and (17) in the CFT description. For this purpose, we assume that the CFT is like a large- $N$ "QCD", i.e., $\mathrm{SU}(\mathrm{N})$ gauge theory with some "quarks".

Before considering couplings of fermions, as a warm-up, we begin with coupling of Higgs to gauge $\mathrm{KK}$ mode (see, for example, 23]). On $5 D$ side, this coupling $\approx g \sqrt{2 k \pi r_{c}} \approx \sqrt{2 g_{5 D}^{2} k}$ [see Eqs. (65) and (60)]: for simplicity, we omit the three SM gauge groups indices in the following). Since these are three particles all of which are localized near $\mathrm{TeV}$ brane, in CFT picture, this is a coupling of 3 composites. We will use the result of a large- $N$ QCD theory in

by $\gamma-\rho$ mixing.

${ }^{11} c=1 / 2$ corresponds to marginal coupling just like $A_{\mu} J_{C F T}^{\mu}$. 
which naive dimensional analysis (NDA) estimation yields:

$$
\text { coupling of } 3 \text { composites } \sim \frac{4 \pi}{\sqrt{N}}
$$

(see, for example, reference [25]). With a coupling of this size, loops are suppressed by $\sim 1 / N$ compared to tree-level.

Assuming duality, we equate the above two couplings to obtain the following relation between $N$ (number of colors of CFT) and parameters of the $5 D$ theory

$$
\sqrt{g_{5 D}^{2} k} \sim \frac{4 \pi}{\sqrt{N}}
$$

Is there a consistency check of this relation? The answer is yes by comparing low-energy gauge coupling on the two sides (see 6th reference of [3]). On the CFT side, we get

$$
1 / g^{2} \sim N /\left(16 \pi^{2}\right) \log (k / \mathrm{TeV}) .
$$

This is due to contributions of CFT quarks to running of external gauge couplings from the Planck scale down to the TeV scale (just like contribution of SM quarks to running of $\left.\alpha_{Q E D}\right)$; whereas, using $\log (k / \mathrm{TeV}) \sim k \pi r_{c}$, we can rewrite the zero-mode (i.e., low energy) gauge coupling on $5 D$ side (see Eq. (60) ) as

$$
1 / g^{2}=\log (k / \mathrm{TeV}) /\left(g_{5 D}^{2} k\right)
$$

These two gauge couplings agree using the relation in Eq. (22) ${ }^{12}$. In particular, we see that $N \sim 5-10$ is required to get $O(1)$ low-energy gauge coupling.

We now move on to couplings of fermions which will give us a semi-quantitaitive understanding of flavor structure using the CFT picture. Begin with couplings of fermions to Higgs. The coupling of $2 \mathrm{KK}$ fermions to Higgs is $\approx 2 \lambda_{5 D} k$ and replacing $\mathrm{KK}$ mode (localized near TeV-brane) in this coupling by zero-mode fermion we get a suppression in $5 D$ picture (due to wavefunction at $\mathrm{TeV}$ brane of zero-mode vs. KK mode) of $\sim 1 / f_{x^{i}}$ (see Eqs. (8) and (177)).

In the CFT picture, the coupling of $2 \mathrm{KK}$ fermions and Higgs (again, three particles localized near TeV brane) is a coupling of 3 composites. Also, coupling of SM/physical fermion to composite Higgs must involve its composite component, i.e., we have to pay the price of mixing $\equiv \xi_{x^{i}}$ between fundamental fermion and CFT composite each time we replace a KK fermion by a zero-mode/SM fermion in the above coupling. So, we get (for example, for $Q$ and $d$ modes)

$$
\mathcal{L}_{0, K K}^{Y} \sim h \frac{4 \pi}{\sqrt{N}}\left(\psi_{Q}^{m} \psi_{d}^{n}+\xi_{Q} \psi_{Q}^{0} \psi_{d}^{n}+\xi_{d} \psi_{d}^{0} \psi_{Q}^{n}+\xi_{Q} \xi_{d} \psi_{Q}^{0} \psi_{d}^{0}\right)
$$

\footnotetext{
${ }^{12}$ Here, we have assumed that the gauge coupling in the CFT picture has a Landau pole at the Planck scale - this is dual to small Planck brane kinetic terms on RS1 side (see 6th reference of 3 and 23 ).
} 
Assuming duality, we equate Eq. (24) and Eqs. (8) and (17) to obtain ${ }^{13}$

$$
2 \lambda_{5 D} k \sim \frac{4 \pi}{\sqrt{N}}
$$

and

$$
\xi_{Q, d} \sim \frac{1}{f_{Q, d}}
$$

Using $N \sim 5-10$ (obtained before), we get $2 \lambda_{5 D} k \sim 5$ - this size of $\lambda_{5 D} k$ agrees with the one before (based on top Yukawa).

We can check the relation in Eq. (26) using coupling of fermion to gauge KK mode. The coupling of gauge KK mode to $2 K K$ fermions (again, three particles localized near $\mathrm{TeV}$ brane) is similar to gauge KK coupling to Higgs, i.e., in the CFT picture, it is a coupling of 3 composites. As in the case of coupling to Higgs, replacing a KK fermion by zero-mode/physical fermion in this coupling $\operatorname{costs} \xi_{x}^{i}$ in the CFT picture so that we get (for simplicity, we show couplings of $Q$ modes only):

$$
\mathcal{L}_{K K \text { composite }}^{g} \sim \frac{4 \pi}{\sqrt{N}} G^{n}\left(\psi_{Q}^{m} \psi_{Q}^{n}+\xi_{Q} \psi_{Q}^{0} \psi_{Q}^{n}+\xi_{Q}^{2} \psi_{Q}^{0} \psi_{Q}^{0}\right)
$$

These couplings in CFT picture agree with Eq. (16) using Eqs. (22) and (26).

In Eq. (27), we considered the coupling involving composite component of gauge KK mode so that we had to use the composite component of zero-mode fermions as well which cost $\xi$ (KK fermions are mostly composite) and hence the subscript "composite" in Eq. (27). However, the gauge KK mode also has an elementary component since the elementary gauge field mixes with spin1 composites ( $\rho$-mesons). It turns out that gauge field is like a fermion with $c=1 / 2$ (for example, zero-mode of gauge field has flat profile just like a fermion with $c=1 / 2)$ so that mixing of elementary gauge boson with $\rho$-meson is given by (using Eq. (26) and Eq. (10))

$$
\xi_{\text {gauge }} \sim \frac{1}{\sqrt{k \pi r_{c}}}
$$

Then, the elementary component of gauge KK mode gives the following couplings. Here, we have to use elementary component of KK fermion which costs $\sim \xi_{x}^{i}$ (zero-mode fermion is mostly elementary):

$$
\mathcal{L}_{\text {KK elementary }}^{g} \sim g \xi_{\text {gauge }} G^{n}\left(\xi_{Q}^{2} \psi_{Q}^{m} \psi_{Q}^{n}+\xi_{Q} \psi_{Q}^{0} \psi_{Q}^{n}+\psi_{Q}^{0} \psi_{Q}^{0}\right),
$$

where $g$ is coupling of elementary gauge boson. The last coupling in Eq. (29) agrees with flavor-independent coupling in Eq. (16) (using Eq. (22)), whereas

\footnotetext{
${ }^{13}$ Since, in the CFT picture, mixing depends on anomalous dimension of fermionic operator and, on RS side, $f_{x}^{i}$ depends on $c$ parameter, using Eq. [26], we obtain a relation between $c$ parameter (i.e., $5 D$ mass of fermion) and the anomalous dimension of fermionic operator which agrees with the standard AdS/CFT dictionary (see, for example, 24).
} 
1st and 2nd couplings in Eq. (29) are too small and hence were not shown in Eq. (16).

To summarize, in the CFT picture the factor of $1 / f$ each time a zero-mode couples to gauge/fermion KK modes or Higgs (apart from universal coupling to gauge KK mode) is due to mixing of fundamental and composite fermions. This mixing is required in order for physical fermion (which is the resultant of this mixing) to couple to composites of CFT (i.e., KK modes/Higgs). Thus, we see that even semi-quantitatively, the small flavor-dependence in coupling to gauge KK modes is correlated with small Yukawa coupling to Higgs.

\section{Signals}

In the previous parts we focused on studying the general structure of the flavor sector of our framework. We found that there is an organizing principle that allows for a transparent understanding of the structure of the flavor violating interactions. We are now at a point at which we can discuss the phenomenological implications of the above analysis.

Before going into the details we shall anticipate which class of FCNC processes might be sensitive to NP contributions. With $m_{\mathrm{KK}} \sim 3 \mathrm{TeV}$ and the approximate flavor symmetries for the light quarks (see subsection [3.3) NP contributions cannot, in general, compete with SM tree level ones. The same conclusion holds for processes which in the SM are mediated by QCD penguin diagrams, e.g. $B \rightarrow \phi K_{S}$ as briefly discussed in 5.2 This is related to the fact that flavor diagonal couplings between light fermions and a KK gluon is given by $\frac{g}{\sqrt{k \pi r_{c}}}$ (see Eq. (16)) so that it is suppressed by $O(5)$ compared with naive expectation.

Consequently we shall focus below on three classes of FCNC processes which receive sizable contributions in the presence of low KK masses, $m_{\mathrm{KK}} \lesssim 3 \mathrm{TeV}$. $\Delta F=2$ processes induced by KK gluon exchange, $\Delta F=1$ processes induced by a shift in the $Z$ couplings and radiative B decays which are enhanced due to large $5 D$ Yukawa (required to obtain $m_{t}$ ) combined with large mixing angles in the right handed down type rotation matrix $D_{R}$. Finally, we shall discuss the model predictions related to EDMs which are sensitive to flavor diagonal CP phases.

\section{1 $\Delta F=2$ processes and the "coincidence problem"}

We start by considering the class of $\Delta F=2$ FCNC processes. These are mediated through tree exchange of KK gluon as shown in figure 1. The contributions were already considered in [6] but it was done for the case with the little hierarchy, i.e., with $m_{\mathrm{KK}} \gtrsim 10 \mathrm{TeV}$ which suppresses FCNC (in addition to the built-in approximate symmetries and the RS-GIM mechanism). Consequently, no large effect was found.

Given the couplings between the zero modes and the KK gluons (16) it is straightforward to estimate the size of the NP contribution. In terms of spurions 
the leading NP contribution to the $B^{0}-\bar{B}^{0}$ mixing amplitude, $M_{12}^{\mathrm{RS}}$, in the mass basis is proportional to

$$
\begin{aligned}
M_{12}^{\mathrm{RS}} & \propto\left[\left(F_{Q} F_{Q}^{\dagger}\right)_{13}\right]^{2} \approx\left[\sum_{i}\left(D_{L}^{\dagger}\right)_{1 i} f_{Q^{i}}^{-2}\left(D_{L}\right)_{i 3}\right]^{2} \\
& \sim\left[\left(D_{L}^{\dagger}\right)_{13} f_{Q^{3}}^{-2}\left(D_{L}\right)_{33}\right]^{2} \sim C_{B}\left|V_{t b}^{*} V_{t d}\right|^{2} f_{Q^{3}}^{-4}
\end{aligned}
$$

where $D_{L}$ is a rotation matrix of the down type, left handed, quarks and $C_{B}$ is an order one complex number. Similar contributions proportional to $f_{Q^{3}}^{-2} f_{d^{3}}^{-2}$ and $f_{d^{3}}^{-4}$ are subleading due to the smallness of $f_{d}^{3}$ (see table 3) and are therefore omitted above. We find that magnitude-wise the suppression due to flavor violation is similar to the SM case. To estimate the size of the NP contribution we present its value normalized by the SM one ${ }^{14}$

$$
\frac{M_{12}^{\mathrm{RS}}}{M_{12}^{\mathrm{SM}}} \sim \frac{16 \pi^{2}}{N_{c}} \frac{8 g_{s}^{2}}{g_{2}^{4} S_{0}\left(m_{t}\right)} \frac{M_{W}^{2}}{m_{\mathrm{KK}}^{2}} \frac{k \pi r_{c}}{f_{Q^{3}}^{4}} \sim 0.5 \times\left(\frac{3 \mathrm{TeV}}{m_{\mathrm{KK}}}\right)^{2}\left(\frac{3}{f_{Q^{3}}}\right)^{4},
$$

where $1 / N_{c}=3$ suppression stems from the contraction of the two octet operators from the two gluonic vertices and $S_{0}\left(m_{t}\right) \sim 2.5$ comes from computing the SM box diagram (See e.g. [26] and refs. therein).

From eq. (31) we learn that with $m_{\mathrm{KK}} \lesssim 3 \mathrm{TeV}$ even with $f_{Q^{3}}$ near to its maximal value the NP contributions to $\Delta F=2$ processes are of the same size as the SM ones. Furthermore since the above NP contributions come with an arbitrary phase, [appears in $\left(D_{L}\right)_{33}^{*}\left(D_{L}\right)_{31}$ ] we expect also an order one contributions to processes such as, $S_{B \rightarrow \psi K_{S}}$ and $S_{B \rightarrow \rho \rho}$, the CP asymmetries in $B \rightarrow \psi K_{S}, \rho \rho^{15}$, which, in the SM, measures the value of $\sin (2 \beta)$ and $\sin (2 \alpha)$ respectively. In addition, a similar derivation yields also sizable contributions to the imaginary part of $\Delta S=2$ processes, This implies that $\varepsilon_{K}$ also receives NP contributions comparable with the SM ones

$$
\begin{aligned}
\varepsilon_{K}^{\mathrm{RS}} & \propto \operatorname{Im}\left[\left(F_{Q} F_{Q}^{\dagger}\right)_{12}\right]^{2} \approx \operatorname{Im}\left[\sum_{i}\left(D_{L}^{\dagger}\right)_{1 i} f_{Q^{i}}^{-2}\left(D_{L}\right)_{i 2}\right]^{2} \\
& \sim \operatorname{Im}\left[\left(D_{L}^{\dagger}\right)_{13} f_{Q^{3}}^{-2}\left(D_{L}\right)_{23}+\left(D_{L}^{\dagger}\right)_{12} f_{Q^{2}}^{-2}\left(D_{L}\right)_{22}\right]^{2} \\
& \sim C_{\varepsilon}\left|V_{t d}^{*} V_{t s}\right|^{2} f_{Q^{3}}^{-4}
\end{aligned}
$$

where $C_{\varepsilon}$ is an order one parameter and note that both of the contributions from $f_{Q^{2}}^{-2}$ and from $f_{Q^{3}}^{-2}$ are of similar size. It is clear that (31) also holds in this case

$$
\frac{\varepsilon_{K}^{\mathrm{RS}}}{\varepsilon_{K}^{\mathrm{SM}}} \sim 0.5 \times\left(\frac{3 \mathrm{TeV}}{m_{\mathrm{KK}}}\right)^{2}\left(\frac{3}{f_{Q^{3}}}\right)^{4}
$$

\footnotetext{
${ }^{14}$ We use $m_{\mathrm{KK}} \sim 3 \mathrm{TeV}$ as favored by the Higgs models. The Higgsless models favors smaller KK masses which will further enhance the NP contributions.

${ }^{15}$ for more information on $S_{B \rightarrow \rho \rho}$ see 27 and refs. therein.
} 
Finally similar results are also obtained for the NP contributions related to $\Delta m_{s}$, the mass difference between $B_{s}^{0}$ and $\bar{B}_{s}^{0}$.

Consequently, the framework predicts sizable $\mathrm{CP}$ asymmetry in (e.g.)

$$
B_{s} \rightarrow \psi \phi
$$

$$
S_{B \rightarrow \psi \phi} \sim 1 \times\left(\frac{2 \mathrm{TeV}}{m_{\mathrm{KK}}}\right)^{2}\left(\frac{3}{f_{Q^{3}}}\right)^{4},
$$

where the SM prediction is $S_{B \rightarrow \psi \phi} \sim \mathcal{O}\left(\lambda^{2}\right)$.

Before studying the implications of these NP contributions, we point out that in reference [19], smaller values of $f_{Q^{3}}$ were considered such that the constraint from $Z \rightarrow b \bar{b}$ is not satisfied. This leads to larger effect in $\Delta F=2$ processes. In particular, to suppress the NP contribution to $\Delta m_{B_{d}}$ requires $\left(D_{L}\right)_{13} \ll V_{t d}$ which is possible only if there are hierarchies in the $5 D$ Yukawa, a possibility that we are not entertaining in this work.

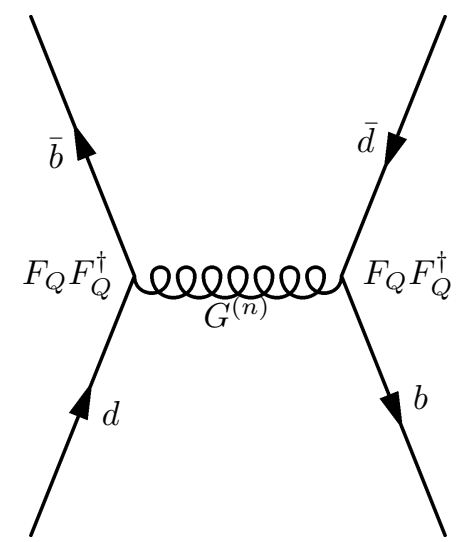

Fig. 1: Contributions to $\Delta F=2$ processes from KK gluon exchange.

\subsection{1 "Coincidence" problem}

Within the SM, experimental data related to the above observables is translated into constraints on, $\rho$ and $\eta$, the less constrained Wolfenstein parameters. The other two parameters, $A$ and $\lambda$ are known to a good accuracy from various SM tree level processes which are insensitive to our NP contributions. The fact that the SM can successfully fit, within errors, five independent measurements of $\rho, \eta$ supports the SM CKM paradigm [28, 29, 30]. Within our framework, however, this SM successful fit is a pure coincidence! This is since the above processes receive uncorrelated sizable NP contributions. Thus, generically, it is not expected that all of them can be fitted together by only two parameters.

In fig. 2. we show the present SM fit yielded by $\Delta F=2$ processes in the $\rho-\eta$ plane 31 32. Due to hadronic uncertainties the "coincidence problem" is yet not a severe one 33. In the near future, assuming that the SM fit will 
continue to be a successful one, when various uncertainties are expected to be brought down and more measurements will be made the problem will be sharpened. This is illustrated by fig. 3. which shows the $\rho-\eta$ plane in the presence of various new constraints (assuming NP contributions are negligible) from processes which may become feasible to experiments in the future [34].

The interesting aspect of this coincidence problem is that it leads to signals! Since the natural size of NP contribution to $\Delta F=2$ processes is comparable to SM, it is clear that the fit to data in this NP model requires $\rho, \eta$ which are $O(1)$ different than in the SM fit. This implies that the angle $\gamma$ in this model is also different than in the SM fit. Thus, $\mathrm{CP}$ asymmetries in $B \rightarrow \rho \rho, D K$ which are measurements of $\gamma$ (after subtracting the mixing phase using $B \rightarrow J / \psi K_{s}$ ) even in the presence of NP (since NP contribution to the decay amplitude is very small) will deviate by $O(1)$ from SM expectations. The preliminary measurements of $B \rightarrow \rho \rho, D K$ seem to agree with SM expectation [35, 36] and thus constrain NP contributions of this size, but the experimental errors are still large.

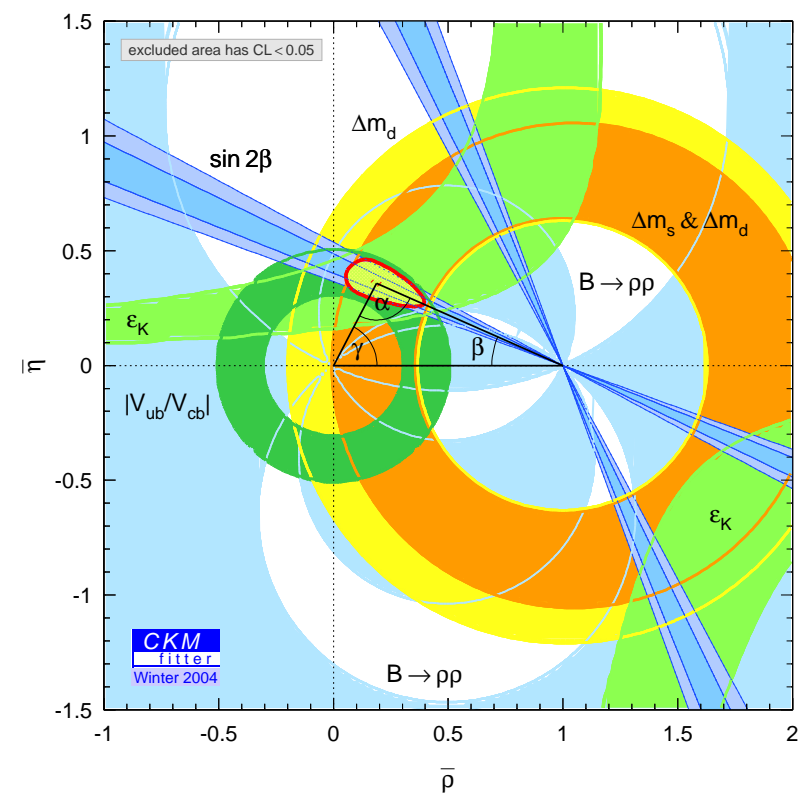


Fig. 2: Current constraints on the unitary triangle 32.

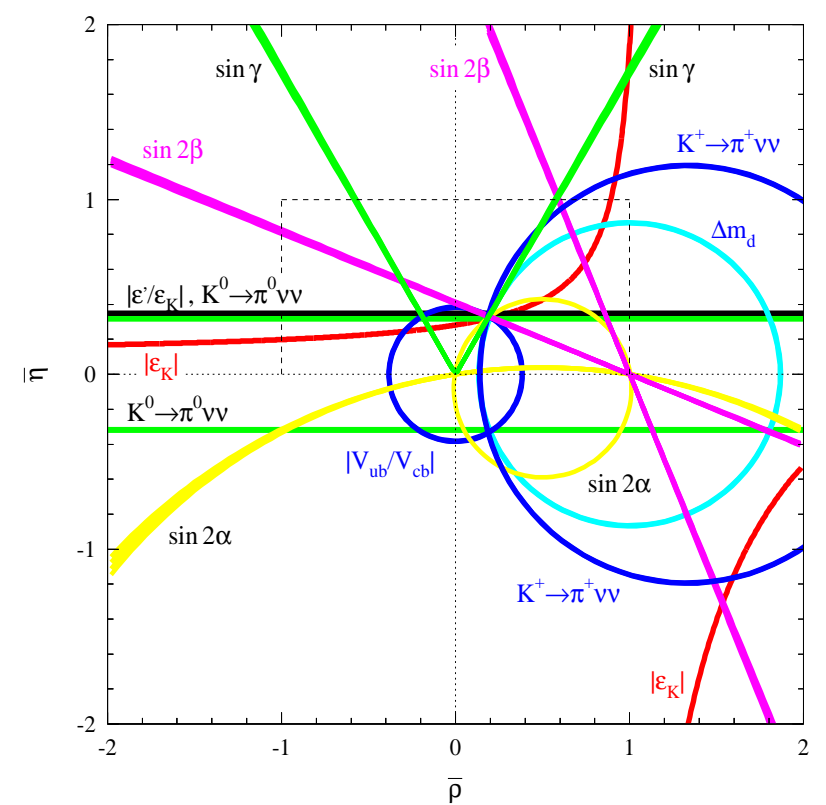

Fig. 3: Illustration: future constraints on the unitary triangle, assuming SM [34].

\section{$5.2 \Delta F=1$ processes}

It is instructive to divide the $\Delta F=1 \mathrm{FCNC}$ processes into two classes. The first proceeds mainly through QCD penguin diagrams e.g. $B \rightarrow K^{0} \bar{K}^{0}, \phi K_{S}$ etc. The second is mediated through electroweak penguin such as semi-leptonic (i.e. $X l^{+} l^{-}$) decays and $\varepsilon^{\prime} / \varepsilon$. We show below that NP contributions to processes from the first class are subleading. NP contribution to the second class of processes are however comparable with the SM ones.

Let us estimate the ratio between the NP and the SM contributions for processes of the first class. The leading NP contributions come from two sources. The first is due to exchange of KK gluons discussed above in the context of $\Delta F=2$ processes. As an example we compare the $b \rightarrow s \bar{s} s \mathrm{NP}$ contribution to the SM one. In term of spurions the leading NP contribution to this process is proportional to (16)

$$
\begin{aligned}
A_{b \rightarrow s \bar{s} s}^{\mathrm{RS}} & \propto\left(F_{Q} F_{Q}^{\dagger}\right)_{23} \approx \sum_{i}\left(D_{L}^{\dagger}\right)_{2 i} f_{Q^{i}}^{-2}\left(D_{L}\right)_{i 3} \\
& \sim C_{s}\left|V_{t b}^{*} V_{t s}\right| f_{Q^{3}}^{-2}
\end{aligned}
$$

where $C_{s}$ is an order one complex number and as in the above contribution from the $\mathrm{RH}$ sector, proportional to $f_{d^{i}}^{-2}$, are further suppressed. The ratio between 
the NP and the SM contribution is roughly given by

$$
\frac{A_{s \bar{s} s}^{\mathrm{RS}}}{A_{s \bar{s} s}^{\mathrm{SM}}} \sim \frac{16 \pi^{2}}{E_{0}\left(m_{t}\right)} \frac{2}{g_{2}^{2}} \frac{M_{W}^{2}}{m_{\mathrm{KK}}^{2}} f_{Q^{3}}^{-2} \sim 0.1\left(\frac{3 \mathrm{TeV}}{m_{\mathrm{KK}}}\right)^{2}\left(\frac{3}{f_{Q^{3}}}\right)^{2},
$$

where $E_{0}\left(m_{t}\right) \sim 0.3$ comes from computing the penguin diagram [26]. The reason for the smallness of the NP contribution stems from the fact that the flavor diagonal piece in the KK gluon coupling yields a suppression of $1 / \sqrt{k \pi r_{c}} \sim$ 0.15 [see (16)]. ${ }^{16}$ There is an additional contribution (which is further suppressed) from the shift in the $Z$ couplings (18) which yields $\frac{A_{s s}^{\mathrm{RS}}}{A_{s \bar{s}}^{\mathrm{SH}}} \sim \frac{g_{2}^{2}}{g_{s}^{2}} \sim 0.2$.

Furthermore, one should have in mind the fact that the above calculation was done at the EWSB scale. When applying the evolution from the weak scale down to the the $m_{b}$ scale one finds that most of the contribution to the corresponding Wilson coefficients is actually due to mixing with the tree level operators 26]. Therefore, to have a sizable NP effect one actually requires the ratio in (36) to be of $\mathcal{O}(10)$ (see e.g. 37). The conclusion from the above discussion is that the NP contribution cannot compete with processes which, within the SM, are induced by $\Delta F=1 \mathrm{QCD}$ penguin diagrams.

We shall now move to discuss a class of processes which in the SM arise only in the presence of electroweak penguin and box diagrams. Examples of such processes are semileptonic $B, K \rightarrow X_{s, d} l \bar{l}, B, K \rightarrow l \bar{l}, \varepsilon^{\prime} / \varepsilon$. We expect that NP contributions from shift of the coupling to the $Z$ will be comparable to the $\mathrm{SM}$ ones for processes in the above class. We choose to focus on $B \rightarrow X_{s} l^{+} l^{-}$ and $K \rightarrow \pi \nu \bar{\nu}$ and show that indeed they receive sizable NP contribution which might be measured in the near future.

We start with $b \rightarrow s l^{+} l^{-}$, in this case following the literature 38, where we parameterize the contributions in term of the effective $Z$ flavor violating couplings, $Z_{s b}^{L}$. In term of spurions the leading NP contribution is proportional to (18)

$$
\begin{aligned}
Z_{s b}^{L \mathrm{RS}} & \propto \delta g_{Z}\left(F_{Q} F_{Q}^{\dagger}\right)_{23} \approx \delta g_{Z} \sum_{i}\left(D_{L}^{\dagger}\right)_{2 i} f_{Q^{i}}^{-2}\left(D_{L}\right)_{i 3} \\
& \sim \delta g_{Z} C_{z}\left|V_{t b}^{*} V_{t s}\right| f_{Q^{3}}^{-2}
\end{aligned}
$$

where $C_{z}$ is an order one complex number and contributions to the coupling of the other chirality, $Z_{s b}^{R \mathrm{RS}}$, are subdominant. The ratio between the NP and the $\mathrm{SM}$ contribution is then roughly given by

$$
\frac{Z_{s b}^{L \mathrm{RS}}}{Z_{s b}^{L \mathrm{SM}}} \sim \frac{4 \pi^{2}}{g_{2}^{2} C_{10}^{*}} \frac{M_{Z}^{2}}{m_{\mathrm{KK}}^{2}} \frac{k \pi r_{c}}{f_{Q^{3}}^{2}} \sim 0.4 \times\left(\frac{3 \mathrm{TeV}}{m_{\mathrm{KK}}}\right)^{2}\left(\frac{3}{f_{Q^{3}}}\right)^{2},
$$

where $C_{10}^{*} \sim 1$ is the corresponding Wilson coefficient 38. This implies that within our framework order one deviation for the branching ratio of $b \rightarrow s l^{+} l^{-}$

\footnotetext{
${ }^{16}$ Note that, as mentioned earlier, reference [19] assumed smaller values of $f_{Q^{3}}$ such that the constraint from $Z \rightarrow b \bar{b}$ is not satisfied. So, NP contribution to $\Delta F=1$ from KK gluon exchange can be larger than in our analysis.
} 
from the SM prediction is expected 39, 13. Similar deviation is also expected in the short distance contributions for the corresponding exclusive modes. The current experimental and theoretical uncertainties for the above inclusive branching ratio is of $\mathcal{O}(30 \%)$ and $\mathcal{O}(20 \%)$ respectively [38. It is clear that the experimental statistical error is going to decrease in the near future. The above process therefore will yield an important test for the framework.

Additional piece of non-trivial information can be extracted by measurement of the lepton forward-backward asymmetry and spectrum of leptons [39. As the new physics (see Eq. 387) contributes mostly to the axial part of the lepton pair current it is expected to yield a sizable modification to the angular distribution of the outgoing leptons accompanied by a vector strange meson. This implies modification of the location of the zero in the low $q^{2}$ region and the value of the integrated asymmetry for the high $q^{2}$ range 38 .

Note that the NP contribution has, in general, a weak phase not related to SM contribution and also NP contribution has no strong phase from real intermediate states (unlike the SM penguin diagram) since it is a tree-level effect. Thus, $O(1)$ direct $\mathrm{CP}$ asymmetry is expected in this process.

Similar flavor violating $Z$ coupling contributes also to the $K^{+} \rightarrow \pi^{+} \nu \bar{\nu}$ decay process where for this case we focus on the $Z s d$ coupling, $Z_{s d}^{L} \mathrm{RS}$. In term of spurions we find

$$
\begin{aligned}
Z_{s d}^{L \mathrm{RS}} & \propto \delta g_{Z}\left(F_{Q} F_{Q}^{\dagger}\right)_{12} \approx \delta g_{Z} \sum_{i}\left(D_{L}^{\dagger}\right)_{1 i} f_{Q^{i}}^{-2}\left(D_{L}\right)_{i 2} \\
& \sim \delta g_{Z}\left[\left(D_{L}^{\dagger}\right)_{13} f_{Q^{3}}^{-2}\left(D_{L}\right)_{23}+\left(D_{L}^{\dagger}\right)_{12} f_{Q^{2}}^{-2}\left(D_{L}\right)_{22}\right] \\
& \sim \delta g_{Z} C_{z}^{\prime}\left|V_{t d}^{*} V_{t s}\right| f_{Q^{3}}^{-2}
\end{aligned}
$$

where $C_{z}^{\prime}$ is an order one complex number. The ratio between the NP and the $\mathrm{SM}$ contribution is then roughly given by

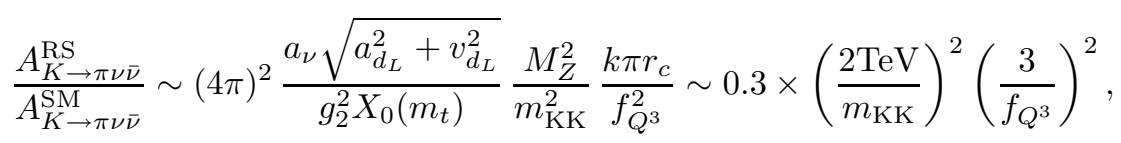

where $X_{0}\left(m_{t}\right) \sim 1.6$ is the corresponding Wilson coefficient 38 . Thus we find a sizable NP contribution uncorrelated with the SM one. The theoretical accuracy for the SM prediction for the $\mathrm{BR}\left(K^{+} \rightarrow \pi \nu \nu\right)$ is around $5-15 \%$ 40, 41. The recent experimental result in 42 already probes the interesting region in the model parameter space in which the SM and the NP constructively interfere (though the future status of the experiment is not clear. For recent work see 43 . and refs. therein). Indeed the central value of the experimental result [42] is considerably higher than the SM prediction [40, 29] which could be an indication of NP; however the errors are quite large at this point to draw a firm conclusion. It is clearly very important to improve this experimental determination.

Indeed NP due to WED has even more striking implication for $K_{L} \rightarrow \pi^{0} \nu \nu$. First recall that this process is theoretically extremely clean as the rate in the $\mathrm{SM}$ is $\mathrm{CP}$ violating and this is a very nice way to measure $\eta$ which drives the $\mathrm{CP}$ 
violation in the CKM-paradigm 28]. Therefore, it is overwhelmingly dominated by the top quark. The presence of the complex coefficient in eq. 40], and therefore a new CP odd phase, can also contribute in our framework to $K_{L} \rightarrow \pi^{0} \nu \nu$ and cause a significant deviation from the prediction of the SM. These very difficult $K_{L}$ experiments 44 then become a very nice way to constrain the phase due to NP. The possible presence of NP source of CP in K-decays due to WED should give an additional impetus to a separate determination of the unitarity triangle purely from K-physics [4].

Using eqs. 16/17/18 it is straight forward to apply the above analysis to other similar $\Delta F=1$ electroweak processes. We shall not elaborate on the others since we hope that the procedure is rather transparent. For recent overview on the current experimental and theoretical status of other related processes see e.g. [46] and refs. therein.

\subsection{Dipole operators}

The class of FCNC processes related to radiative B decays provides a lot of information on the structure of the effective theory at and above the EWSB scale. Measurements of the $\mathrm{BR}(b \rightarrow s \gamma)$ already provide a powerful constraint on NP models. SM contributions induce, to leading order, single chirality operators $O_{7 \gamma}$ and $O_{8 g}$ with

$$
O_{7 \gamma, 8 g}=\bar{b}_{R} \sigma^{\mu \nu} s F^{\mu \nu}, G^{\mu \nu},
$$

where $F^{\mu \nu}$ and $G^{\mu \nu}$ are the field strengths for the electromagnetic and chromomagnetic interactions. In the SM, the Wilson coefficients $C_{7 \gamma, 8 g}^{\prime}$ of the opposite chirality operators, $O_{7 \gamma, 8 g}^{\prime}$, are suppressed by $m_{s} / m_{b}$ and therefore are negligible (or $m_{d} / m_{b}$ for the $b \rightarrow d \gamma$ processes). The fact that there are no right handed chiral operators is a unique feature to the SM which will be tested in the near future as discussed below. We therefore focus on the NP contributions for the above processes. We show that in our framework the value of $C_{7 \gamma, 8 g}^{\prime}$ is found to be comparable with that of the SM contribution to $C_{7 \gamma, 8 g}$.

From eqs. (16/17/18) we find that there are no tree level flavor changing contributions which induce helicity flip as required by the above process. Consequently, we now discuss loop processes which involve KK states in the loop. Contribution from an individual KK state is finite but the sum is logarithmically sensitive to the cutoff. This can be understood from the fact that the contribution must involve the $\mathrm{TeV}$ brane (in the model with EWSB localized on $\mathrm{TeV}$ brane) to account for the helicity flipping. This softens the UV sensitivity: brane-localized interactions have higher degree of divergence than bulk interactions. Consequently in the 5D Lagrangian there is a counter term which cancels the above log divergence. Generically, without fine tuning, the finite loop contribution is not expected to be exactly canceled by the counter term ${ }^{17}$. Thus our calculation below just estimates the size of the finite UV insensitive $\mathrm{NP}$ contribution. We also mention that with the low cutoff at the TeV brane,

\footnotetext{
${ }^{17}$ As expected, the NDA estimate for contribution from cut-off physics (which can be at tree-level) is comparable to the calculable loop contribution: see section 5.4
} 
the logarithm is not expected to be large and therefore, is, in principle, under control. As expected, with Higgs in bulk (but with wavefunction peaked near $\mathrm{TeV}$ brane) [17, the loop contribution is finite since the helicity flip is no longer a brane-localized interaction and the cut-off physics contribution is smaller (see section [5.4).

We can divide the leading NP contribution into three classes:

(i) Flavor violating contributions due to zero mode couplings to KK gluons (shown in figure 4). These contributions are found to be subleading.

(ii) Flavor violating contributions due to zero mode couplings to down type fermion KK modes which requires EWSB (shown in figure 5). These contributions are sizable even in the limit $\lambda_{u 5 D} \rightarrow 0$.

(iii) Flavor violating contributions due to zero mode couplings to down and up type fermion KK modes which requires EWSB (shown in figure 6). These contributions are sizable but require non-zero up and down type Yukawa couplings.

Naively, it is not expected that loop processes with internal heavy KK fields would yield sizable contributions. The reason that we do find sizable contributions for the right handed operators stems from the fact that the entries of $D_{R}$ are all of order unity (see table 3) ${ }^{18}$ which overcomes the corresponding CKM suppression in the SM contribution. Below, the contributions (i,ii,iii) are discussed in that order.

We start by showing why the contribution with KK gluons in the loop to $C_{7 \gamma}^{\prime}, C_{7 \gamma}^{\prime G^{\mathrm{KK}}}$ are small. The relevant diagram is shown in fig. 4. In term of spurions it is proportional to

$$
C^{\prime}{ }_{7 \gamma}^{G^{K K}} \propto\left(F_{Q} \lambda_{d 5 D} F_{d}\right)_{32} \approx \frac{1}{2 v}\left[\operatorname{diag}\left(m_{d, s, b}\right)\right]_{32}=0,
$$

where in the above we suppressed the flavor indices. The reason why $C^{\prime} G_{7 \gamma}^{\mathrm{KK}}$ vanishes is because, according to our approximation, it is aligned with the $4 \mathrm{D}$ down Yukawa matrix. This is discussed in detail in subsection 3.3.1] [see [20], whereas in appendix $\mathbb{B}$ we consider the deviations from the above result and show that the corrections are indeed small.

\footnotetext{
${ }^{18}$ and couplings of KK fermions to Higgs are enhanced by large $5 D$ Yukawa required, in turn, to obtain top quark mass.
} 


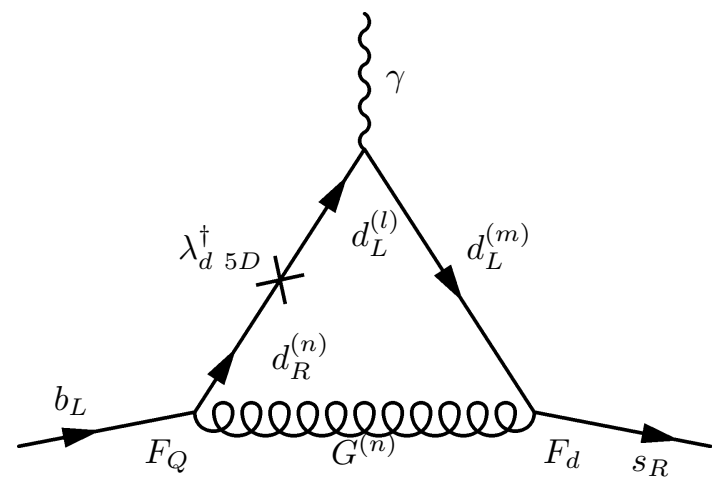

Fig. 4: Contributions to $b \rightarrow s \gamma$ from KK gluon in the loop.

We next move to discuss the contributions with KK fermions in the loop (ii) +(iii). In term of spurions they are given by

$$
C_{7 \gamma}^{\prime} \propto\left[F_{Q}\left(\lambda_{u 5 D} \lambda_{u 5 D}^{\dagger}+\lambda_{d 5 D} \lambda_{d 5 D}^{\dagger}\right) \lambda_{d 5 D} F_{d}\right]_{32},
$$

where the first (second) term in the parenthesis comes from computing the diagram in fig. 6 (fig. 5).

Before continuing we note that in the Higgsless case there are similar contributions as follows: the Higgs line should be replaced by a longitudinal $W, Z$ line which is $W_{5}, Z_{5}$ in the Higgsless models. In addition, just as in the model with the Higgs, the presence of the mass term on the $\mathrm{TeV}$ brane yield mixing between SM doublet (singlet) quarks and singlet (doublet) KK quarks. Using this mass mixing, we can show that similar contributions arise also in the Higgsless case.

In appendix C we show that these are not aligned with the 4D Yukawa matrices. Consequently we expect them to yield sizable contributions. In appendices C.1 and C.2 we computed the flavor structure yielded by the diagrams shown in figures 5 and 6 respectively. It turns our that both the contributions from only down type (see Eq. (89)) and the up type (see Eq. (90) internal KK quarks are comparable. We find that both of them are proportional to $m_{b}\left(D_{R}\right)_{32}$

$$
C_{7 \gamma}^{\prime} \propto a_{\gamma}\left(\lambda_{5 D} k\right)^{2} m_{b}\left(D_{R}\right)_{23}
$$

where $a_{\gamma}$ is an order one complex number. In order to estimate the size of the contribution we divide it by the SM contribution, $C_{7 \gamma}^{\mathrm{SM}}$

$$
\frac{C_{7 \gamma}^{\prime}}{C_{7 \gamma}^{\mathrm{SM}}} \sim \frac{M_{W}^{2}}{m_{\mathrm{KK}}^{2}}\left|\frac{\left(D_{R}\right)_{23}}{V_{t b} V_{t s}^{*}}\right| \frac{\left(\lambda_{5 D} k\right)^{2}}{D_{0}^{\prime}\left(m_{t}\right) g_{2}^{2}} \sim 1 \times\left(\frac{3 \mathrm{TeV}}{m_{\mathrm{KK}}}\right)^{2} \frac{\left(D_{R}\right)_{23}}{2},
$$

where $D_{0}^{\prime}\left(m_{t}\right) \sim 0.4\left[26\right.$ and in the above estimate we used $\left(D_{R}\right)_{23} \sim f_{d^{3}} / f_{d^{2}} \sim$ 0.5 (see (12)) and $\lambda_{5 D} k \sim 4$ which was required to obtain the top quark mass 
given the lower bounds on $f_{u^{3}}$ and $f_{Q^{3}}$ as explained earlier. The NP contributions to $C_{7 \gamma, 8 g}$ are obtained by replacing $\left(D_{R}\right)_{23}$ in the above by $\left(D_{L}\right)_{23} \sim V_{t s}$ and hence are suppressed by $\sim \mathcal{O}(1 / 10)$.

The above results imply that within our framework we expect the emitted photon to be affected by operators with both chiralities unlike the SM case. Upcoming results from the B factories will be sensitive to that effect by either measuring the polarization of the outgoing photon 47 or by measuring timedependent CPV effect [48] in exclusive final states such as $B_{d} \rightarrow \gamma K_{s}^{* 0}(\rho, \omega)$ or in $B_{s} \rightarrow \gamma \phi\left(K_{s}^{* 0}\right)$. Indeed, experimental feasibility of this interesting test has recently been demonstrated [4] and improved tests with greater luminosities at B and Super-B factories are eagerly awaited.

Using similar derivation we find the prediction for the opposite chirality Wilson coefficient, $C_{7 \gamma d}^{\prime}$, for the $b \rightarrow d \gamma$ process (91)

$$
\frac{C_{7 \gamma d}^{\prime}}{C_{7 \gamma d}^{\mathrm{SM}}} \sim \frac{M_{W}^{2}}{m_{\mathrm{KK}}^{2}}\left|\frac{\left(D_{R}\right)_{13}}{V_{t b} V_{t d}^{*}}\right| \frac{\left(\lambda_{5 D} k\right)^{2}}{D_{0}^{\prime}\left(m_{t}\right) g_{2}^{2}} \sim 3 \times\left(\frac{2 \mathrm{TeV}}{m_{\mathrm{KK}}}\right)^{2} \frac{\left(D_{R}\right)_{13}}{6} .
$$

In this case the effect is even more dramatic since within the SM the other chirality operator is suppressed by $\mathcal{O}\left(\frac{m_{d}}{m_{b}}\right)$.

It is also useful to note that RS1 contribution to $C_{7 \gamma}$, which is $\sim \mathcal{O}(1 / 10)$ of SM contribution, has, in general, a different weak phase than SM contribution and also does not have a strong phase from real intermediate states (unlike the SM contribution). Hence, the NP can also affect quite appreciably SM predictions, which are fairly precise, for direct $\mathrm{CP}$ asymmetries [50] in inclusive as well as exclusive final states. In particular recall that SM predicts very small $(\approx 0.6 \%)$ asymmetry in $b \rightarrow s \gamma$ transitions which is about an order of magnitude below the current experimental bound [51]. Continued experimental effort at measurment of direct $\mathrm{CP}$ asymmetries in all of the radiative modes is clearly very important.

Note that the NP contribution to $C_{7 \gamma}^{\prime}$ does not interfere with the SM contribution to $C_{7 \gamma}$ in the rate for $b \rightarrow s \gamma$ so that $C_{7 \gamma}^{\prime R S} \sim 1 / 2 C_{7 \gamma}^{S M}$ is sufficient to be consistent with the measured BR since the theoretical/experimental uncertainties are at the level of $\sim \mathcal{O}(10 \%)$ each [52, [51]. 


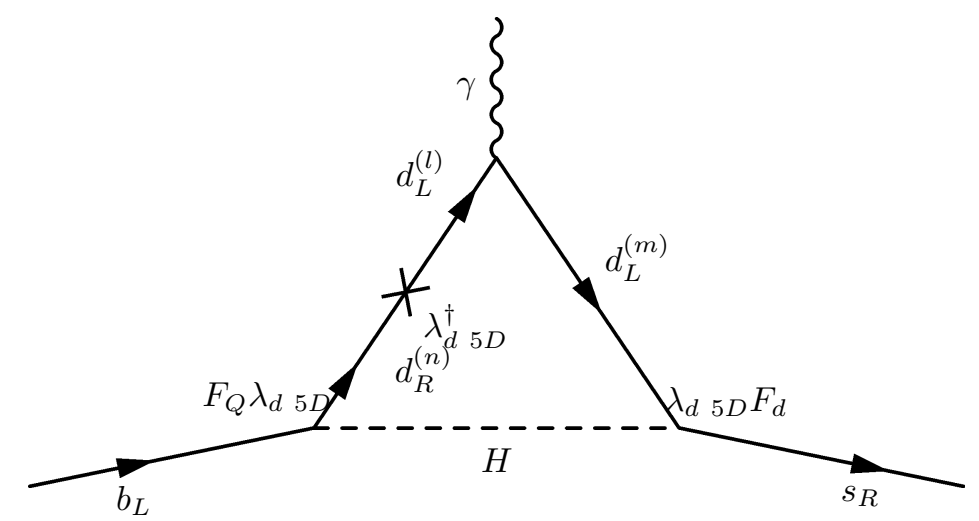

Fig. 5: Contributions to $b \rightarrow s \gamma$ from Yukawa interactions with down type KK quarks.

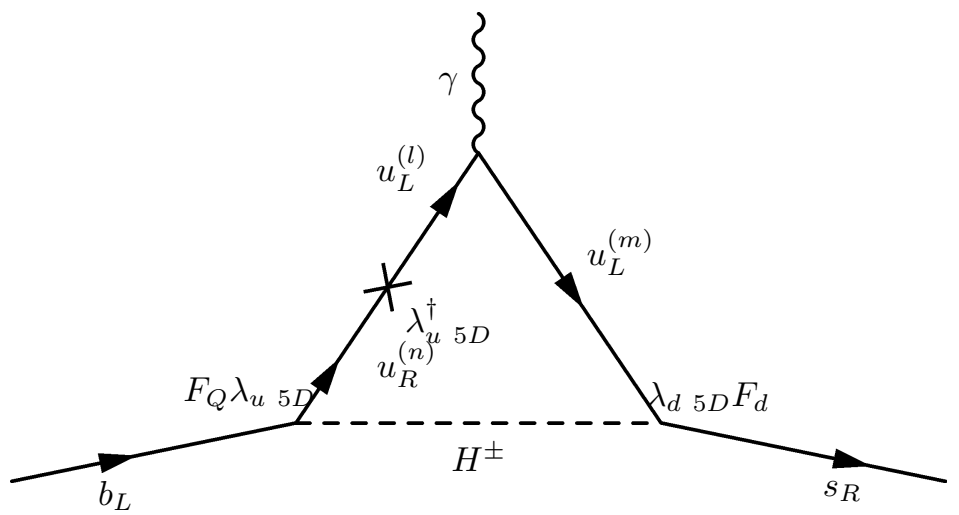

Fig. 6: Contributions to $b \rightarrow s \gamma$ from Yukawa interactions with down and up type KK quarks.

\subsection{Flavor diagonal CPV and electric dipole moments}

It is well known that almost any SM extention contains new sources of CPV. Such sources generically contribute to two classes of CPV observables. The first is related to what we discussed above, that is CPV that occurs through flavor mixing. Several experiments have measured CPV of the above type in the $\mathrm{K}$ and $\mathrm{B}$ systems and it was found to be sizeable. The second class is related to flavor diagonal CPV. This type of CPV has not been observed yet and experimental data yield a severe constraint on the corresponding flavor diagonal CPV sources. This is through measurements of the electron and neutron electric dipole moments (EDMs), $d_{n}$. Current data yield the following bound on the neutron EDM 53

$$
d_{n} \leq 6 \times 10^{-26} \mathrm{e} \mathrm{cm}
$$


In the SM there are two such sources. First, there is the celebrated strong CP phase, $\bar{\theta}$, where the above constraint yields $\bar{\theta} \lesssim 10^{-10}$ (see e.g. [22, 54] and refs. therein). In addition, the CP-odd phase in the CKM matrix [28 can yield a non-vanishing value for the EDM beyond two EW loops [55, 56. However, this is estimated to be extremely small, $\lesssim \mathcal{O}\left(10^{-30} e-\mathrm{cm}\right)$. In SM extensions other sources usually exist which contribute to the EDMs and therefore must be small. A well known example is the supersymmetric CP problem where generically the MSSM predicts the EDMs to be two order of magnitude larger than the current experimental limit (see e.g. 22, 57] and refs. therein).

We find it therefore very important to investigate what is the RS framework prediction regarding the EDMs. Since the structure of the lepton sector is model dependent we chose to focus on the quark sector. The relevant operator, $O_{n}$, which contributes to the EDM is given by

$$
O_{d_{n}}=\bar{d}_{L} \sigma^{\mu \nu} d_{R} F_{\mu \nu}+\text { h.c. },
$$

where EDMs are proportional to the imaginary part of the corresponding Wilson coefficient, $C_{d_{n}}$. As explained in section 5.3 since this process requires helicity flip there is no tree level contributions from KK modes. We shall, therefore, focus on the leading, one-loop, contributions. The required analysis is, actually, similar to the one done above in the context of radiative $B$ decays. In order to estimate $C_{d_{n}}$ we should calculate the contributions from the diagrams shown in figs. 4-6 after we change the external quarks into $d_{L, R}$.

We start by analysing the contibution with internal KK gluons, $C_{d_{n}}^{G^{\mathrm{KK}}}$, from the diagram in fig. 4 . In term of spurions it is given by (41)

$$
C_{d_{n}}^{G^{\mathrm{KK}}} \propto k v\left(D_{L}^{\dagger} F_{Q} \lambda_{d 5 D} F_{d} D_{R}\right)_{11} \approx \frac{1}{2}\left[\operatorname{diag}\left(m_{d, s, b}\right)\right]_{23}=0,
$$

The fact is that the imaginary part of $C_{d_{n}}^{G^{\mathrm{KK}}}$ vanishes because it is approximately aligned with the 4D down Yukwa matrix [see subsection 3.3.1. In appendix B and C.3 we consider the deviations from the above result and show that these are subleading compared to the larger predictions which we discuss below.

We therefore focus on the contributions given by the diagrams in figures 5 and 6 (again changing the external quarks into $d_{L, R}$ ). In term of spurions these are of the form

$$
C_{d_{n}} \propto 2 k^{3} v\left[F_{Q}\left(\lambda_{u 5 D} \lambda_{u 5 D}^{\dagger}+\lambda_{d 5 D} \lambda_{d 5 D}^{\dagger}\right) \lambda_{d 5 D} F_{d}\right]_{11} .
$$

In appendix C.3 we show that $C_{d_{n}}$, generically, contains an unsuppressed imaginary part which cannot be removed by a phase redefinition,

$$
\operatorname{Im}\left(C_{d_{n}}\right) \sim\left|C_{d_{n}}\right| .
$$

Given the above let us estimate what is the model prediction for the EDM

$$
\left(\frac{d_{n}}{e}\right)_{K K} \sim \frac{1}{6} \frac{m_{d}}{16 \pi^{2}} \frac{\left(2 k \lambda_{5 D}\right)^{2}}{m_{K K}^{2}} \sim 10^{-24} \mathrm{~cm}\left(\frac{2 k \lambda_{5 D}}{4}\right)^{2}\left(\frac{3 \mathrm{TeV}}{m_{\mathrm{KK}}}\right)^{2} .
$$


The above result is larger than the experimental bound (46) by a factor of $\mathcal{O}(20)$. This implies that, with $m_{\mathrm{KK}} \lesssim 3 \mathrm{TeV}$, our framework is confronted by a CP problem similar to the SUSY CP problem. Several points are in order regarding the above result (see appendix C.3 for details):

- The imaginary part comes from "Majorana"-like phases and therefore appears already at the two generation level.

- However, with one generation, Majorana phases are absent/not physical so that the contribution does require presence of mixing in both $D_{L}$ and $D_{R}$ and non-degeneracy of both $f_{Q, d}$ 's. This implies the existence of a $\mathrm{CP}$ violating rephase invariant quantity, the analog of the Jarskog determinant of the SM, but originating from Majorana phases, i.e., not from CKM-like phases unlike in the SM. A similar object is discussed in the context of SUSY in the lepton sector 58. In that case a new rephase invariant quantity is found which requires both mixing and non-degeneracy for 2 generation of both left handed and right handed sleptons in order to generate EDM.

- The CKM-like phases cannot contribute to the EDM. This is since the one loop contribution does not involve all the three mixing angles in left or right-handed down sector simultaneously so that we do not have sensitivity to SM-like Jarlskog invariants [58].

- The above contributions are sensitive both to the overall size of the Yukawa couplings and the KK masses. This imply that they decrease faster with larger KK masses than other signals described above. We elaborate more on this point in the conclusions.

In addition there is a contribution from higher-dimensional operator on the $\mathrm{TeV}$ brane. Since this contribution is UV sensitive, we can only estimate it using naive dimensional analysis and compare it with our result in Eq. (51) as follows. We should replace the suppression from the heavy KK mass, $m_{K K}$, by warpeddown cut-off (since operator is on TeV brane), $\Lambda \sim \Lambda_{5 D} e^{-k \pi r_{c}}$, where $\Lambda_{5 D}$ is the (Planckian) $5 D$ cut-off. Furthermore the loop factor $\left(2 \lambda_{5 D} k\right)^{2} /\left(16 \pi^{2}\right)$ is expected to be replaced by an $O(1)$ number. This is since cut-off contribution can be tree-level (unlike ones which come from KK modes). Consequently we find

$$
\left(\frac{d_{n}}{e}\right)_{\Lambda} \sim C_{\Lambda} \frac{m_{d}}{\Lambda^{2}} \sim C_{\Lambda} 10^{-24} \mathrm{~cm}\left(\frac{2 k \lambda_{5 D}}{4}\right)^{2}\left(\frac{10 \mathrm{TeV}}{\Lambda}\right)^{2},
$$

where $C_{\Lambda}$ is an arbitrary complex coefficient.

The cut-off scale is model-dependent:

$$
\begin{aligned}
\Lambda_{\text {brane }} & \sim \frac{4 \pi}{2 \lambda_{5 D} k} m_{K K} \\
& \sim 10 \mathrm{TeV}\left(\frac{4}{2 \lambda_{5 D} k}\right)\left(\frac{m_{K K}}{3 \mathrm{TeV}}\right)
\end{aligned}
$$




$$
\begin{aligned}
\Lambda_{b u l k} & \sim\left(\frac{4 \pi}{2 \lambda_{5 D} k}\right)^{2} m_{K K} \\
& \sim 30 \mathrm{TeV}\left(\frac{4}{2 \lambda_{5 D} k}\right)^{2}\left(\frac{m_{K K}}{3 \mathrm{TeV}}\right),
\end{aligned}
$$

where the subscripts "brane" and "bulk" on $\Lambda$ denote the models with Higgs on $\mathrm{TeV}$ brane 9 and in the bulk 17, respectively Hence, EDMs from cut-off physics are comparable to the naive loop contribution of Eq. (51) so that they exceed experimental limit by $\mathcal{O}(20)$ for Higgs on $\mathrm{TeV}$ brane. On the other hand allowing a rather simple modification of our framework, with Higgs in the bulk (but localized near the $\mathrm{TeV}$ brane) [17, the cut-off effects are comparable to experimental limit and significantly smaller than the contribution induced by figure 5 and 6 given in eq. (51).

\subsection{Flavor violation in up quark sector}

In order to estimate flavor violation in the up type sector we need to consider $U_{L, R}$. Using table 3] and eq. (12), we find that $\left(U_{R}\right)_{12} \sim f_{u^{2}} / f_{u^{1}} \sim O\left(10^{-2}\right)$ is much smaller than $\left(U_{L}\right)_{12}$. Furthermore, $\left(U_{R}\right)_{23} \sim f_{u^{3}} / f_{u^{2}} \sim 0.1$ is somewhat larger than $\left(U_{L}\right)_{23}$ and $\left(U_{R}\right)_{13} \sim 10^{-3}$ is also smaller than $\left(U_{L}\right)_{13}$.

In analogy to $\Delta F=2$ transition in down quark sector, KK gluon exchange gives the following contribution to $D^{0}-\bar{D}^{0}$ mixing [up to $O(1)$ complex coefficients]:

$$
\begin{aligned}
M_{12 L L}^{R S} & \propto\left[\left(U_{L}\right)_{13} f_{Q^{3}}^{-2}\left(U_{L}\right)_{23}\right]^{2}, \\
M_{12 R R}^{R S} & \propto\left[\left(U_{R}\right)_{13} f_{u^{3}}^{-2}\left(U_{R}\right)_{23}\right]^{2}, \\
M_{12 L R}^{R S} & \propto\left[\left(U_{L}\right)_{13} f_{Q^{3}}^{-2}\left(U_{L}\right)_{23}\right]\left[\left(U_{R}\right)_{13} f_{u^{3}}^{-2}\left(U_{R}\right)_{23}\right]
\end{aligned}
$$

where $L L$ denotes contribution to $\left(\bar{u}_{L} \gamma^{\mu} c_{L}\right)^{2}$ operator and so on. The fact that $f_{u^{3}}^{-1}$ is sizeable (in addition to $f_{Q^{3}}^{-1}$ ) results in violation of approximate flavor symmetries/RS-GIM and hence the $L R$ and $R R$ operators are also enhanced (unlike in the down quark case). We compare the NP contribution (dominated by $R R$ operator) to the short distance contribution in SM [59]

$$
\begin{aligned}
\frac{M_{12 R R}^{R S}}{M_{12}^{S M}} & \sim \frac{32 \pi^{2}}{N_{c}} \frac{M_{W}^{2}}{m_{K K}^{2}} \frac{m_{c}^{2} M_{W}^{2}}{\left(m_{s}^{2}-m_{d}^{2}\right)^{2}} \frac{k \pi r_{c}}{f_{u^{3}}^{4}} \frac{g_{s}^{2}}{g^{4}}\left[\frac{\left(U_{R}\right)_{13}\left(U_{R}\right)_{23}}{V_{c s}^{*} V_{c d}}\right]^{2} \\
& \sim 100\left(\frac{3 \mathrm{TeV}}{m_{K K}}\right)^{2}\left(\frac{1.2}{f_{u^{3}}}\right)^{4},
\end{aligned}
$$

where we used $m_{c} \approx 1.2 \mathrm{GeV}$ and $m_{s} \approx 100 \mathrm{MeV}$. We see that the NP contribution is larger than the SM short distance effect by $O(100)$. However, the long distance SM contribution can be larger than the short distance SM contribution by $O(100-1000)$ 60. Also, current experimental limit 61 is still weaker than 
the long distance SM prediction. So, at present there is no constraint on this NP effect using the $D^{0}-\bar{D}^{0}$ mass difference, $\Delta m_{D}$.

On the other hand, the presence of the complex coefficient in eq. 54 means that WED endows the $D^{0}-\bar{D}^{0}$ oscillation a non-standard CP-odd phase. The presence of such a phase should cause time-dependent $\mathrm{CP}$ asymmetries, perhaps $O(10 \%)$, in $D^{0}$ decays which may be cleanly measured via decays to CPeigenstates such as $D^{0} \rightarrow K_{S} \pi^{0}\left(\eta, \eta^{\prime}, \rho \ldots\right)$ or $\phi \pi^{0}$, in complete analogy to $\sin 2 \beta$ measurments via $B^{0} \rightarrow \psi K_{S}$. Note that direct $\mathrm{CP}$ asymmetries in these modes potentially arise through interference of penguin and tree graphs, a la [62] and are expected to be completely negligible since the penguin contribution (inlcuding NP effect) is extremely suppressed. Thus the existing upper bounds of a few percent asymmetries in many such channels 61 are easily understood. This discussion underscores the importance of pursuing time dependent CP studies in charm factories, such as CLEO-c 63.

There are also RS1 contributions to flavor-changing top quark decays, for example, $t \rightarrow c Z$ (analogous to flavor-violating $Z$ vertex involving down quarks) and $t \rightarrow c \gamma$ (analogous to radiative $B$ decays). A new effect in radiative decays is that, as mentioned earlier, $f_{u^{3}}$ for the top quark is quite different than that of all the other up type singlet quarks. Consequently, the wavefunction and spectrum of $t_{R} \mathrm{KK}$ modes is different than that of other KK fermions and our approximation of KK-blind flavor violation breaks down. This results in KK gluon contribution to dipole operators being not aligned with $4 D$ up Yukawa matrix and so it does contribute to $t \rightarrow c \gamma$ (see appendix). Recall that in the SM, due to the large mass of the top quark the GIM-mechanism becomes exceedingly effective so that not only the branching ratios of these decays $(t \rightarrow$ $c Z(\gamma$, gluon $)$ are extremely suppressed 64, 65], all CP asymmetries 66, 67. driven by the CKM paradigm [28] become completely negligible. Since these decays are sensitive to RS1 top quark FC coupling (including effect of new CP-odd phase(s)), their searches are very well motivated. Such decays can be probed at the Tevatron, LHC and a linear collider (LC) - we will leave this analysis for a future study [68] except also to draw brief attention to another unique process, $e^{+} e^{-} \rightarrow \bar{t} c, t \bar{c}$. These are also very clean reactions to study at a LC in search of signatures of RS1 as in the SM their rate is, once again, exceedingly suppressed [69].

\section{Discussion and conclusions}

It is well known that in order to solve the fine tuning problem any new physics (NP) framework is required to introduce new degrees of freedom at a scale close to the electroweak symmetry breaking (EWSB) scale. These new weakscale fields, however, tend to spoil the successful fit of the SM to electroweak precision measurements (EWPM) and to various measurements related to flavor violation. Thus a tension is induced between the SM experimental success and the need to solve the fine tuning problem.

Recently a framework (containing both Higgs and Higgsless models) based 
on warped geometry with bulk custodial symmetry which relaxes the tension related to EWPM was introduced. We focused in this paper on the flavor sector of this framework with light fermions localized near Planck brane to make flavor issues UV-insensitive. We showed that, regardless of the details of the model, it has an underlying organized structure. This framework has a builtin mechanism to suppress NP contribution related to flavor changing neutral currents $(\mathrm{FCNC}) \mathrm{s}$. It turns out that effectively flavor dynamics is controlled only by physics near the TeV brane. This implies that besides the SM Yukawa matrices all the NP flavor violation is governed by three additional spurions $F_{Q, u, d}$ that transform as bi-fundamentals under the SM and the diagonal KK quark flavor group, $\mathrm{U}(3)_{Q, u, d} \times \mathrm{U}(3)_{Q, u, d}^{n}$. These are just related to the value of the zero mode profiles on the $\mathrm{TeV}$ brane. The built in suppression of FCNC stems from the fact that: (i) the entries in $F_{Q, u, d}$ related to the light quarks are small which yield an approximate flavor symmetries. (ii) only the non-universal part in $F_{Q, u, d}$ can induce flavor violation which yield the RS-GIM mechanism. Consequently we find that, as in the SM, flavor violation in the model is sizable due to third generation GIM breaking.

The actual models that we study provide a solution to the SM flavor puzzle. That is the 5D Yukawa matrices are assumed to be anarchical and the hierarchy in the SM flavor parameters is accounted by the split fermion mechanism. This assumption turns the framework into a predictive one and our results become robust and independent within a class of models in this overall framework.

We find that NP could be detected in three classes of FCNC process: (i) $\Delta F=2$ transitions; (ii) $\Delta F=1$, mainly in semi-leptonic decays (e.g. $B \rightarrow$ $X_{s} l^{+} l^{-}$); (iii) Radiative $\mathrm{B}$ decays. In addition we showed the contributions to EDMs from KK states are about an order of magnitude above the current experimental bound for KK masses $\sim 3 \mathrm{TeV}$. Thus there is an RS CP problem.

It is important to note that the contributions related to class (iii) including the EDMs are more sensitive to the assumption that the KK masses are small as follows. This is due to the fact that they are proportional to both the square of the 5D Yukawa couplings and inverse square of the KK masses. If we slightly increase the KK masses (to $\sim 4 \mathrm{TeV}$ ) however the EWPM related to the shift of the coupling of $b_{L}$ to $Z$ are weakened. This implies that $Q_{3}$ can localized more towards the $\mathrm{TeV}$ brane $\left(f_{Q^{3}}\right.$ is smaller) which enhances the top mass. Consequently this allows for a lower (by a factor of $\sim 2$ ) overall scale of the Yukawa couplings. Thus the new physics contributions to radiative B decays and EDMs falls much more rapidly (by a factor of $\sim 10$ for KK mass $\sim 4 \mathrm{TeV}$ ) than naively expected. On the other hand, the NP contributions of classes (i+ii) are proportional to square of $b_{L}$ coupling to gauge KK mode and to the inverse square of the KK masses: the latter increases as $Q_{3}$ is localized closer to the $\mathrm{TeV}$ brane in such a way that these NP effects remain roughly the same for KK mass $\sim 4 \mathrm{TeV}$.

We finally want to comment of how to proceed from this point. Our work is based on the concept that we should test the predictions of the framework allowing for minimal possible fine tuning. This is why we assume that the Yukawa matrices are anarchical and that the KK masses are rather low to 
reduce the little hierarchy.

Future measurements of processes related to items (i)-(iii) might find deviation from the SM prediction supporting the above framework. Two questions are important to ask in advance as follows:

- What if no deviations from SM predictions are found?

We can either just say that the KK masses are actually higher - this implies that the framework requires more fine tuning and therefore becomes less attractive. Other possibility is that it might be that accidentally one or two elements of the 5D Yukawa matrices are smaller than their naive value. This may induce a small value of $\left(D_{L}\right)_{13}$. The consequence of this is accidental suppression of some of the above contributions. We believe that due to correlation between various observables discussed above one will be able to test this hypothesis and verify or falsify this explanation. Analysis of non-trivial correlations between the predictions of this framework is left to future research.

- What if one finds deviation in one of the classes of FCNC processes which are not sensitive to NP contribution within our framework? In this case we claim that the above framework will be disfavored and we do not find a way of accounting for such a situation. A realistic example of such a scenario is data which would signal a large deviation from the SM prediction in processes which in the SM are dominated by tree level or QCD penguin diagram. Leading candidate for that situation is the CP asymmetry in $B \rightarrow \phi K_{S}$.

We also should remark that in our study above we consider the simplest class of model without $\mathrm{TeV}$ brane kinetic terms 70 and with EWSB (with or without Higgs) on the $\mathrm{TeV}$ brane. Note, however, in models with Higgs in the bulk [17, the Higgs profile is still localized near TeV brane. So, our study is valid for these models as well. We should though stress that recent studies of electroweak precision tests (EWPT) in warped Higgsless models [15, 16] suggest that, even in the presence of brane kinetic terms, these models can be consistent with EWPT only if the KK modes (even the 1st one) are strongly coupled. This implies the presence of new physics at the KK mass scale (related to the cut-off) leads to loss of predictivity since the NDA size of (uncalculable) cut-off effects are comparable to (or in the case of radiative effects, larger than) the KK effects which we studied.

\section{Acknowledgements}

KA is supported by the Leon Madansky fellowship and NSF Grant P420D3620414350; GP is supported by the Director, Office of Science, Office of High Energy and Nuclear Physics of the US Department of Energy under contract DE-AC0376SF00098. Research of AS is supported in part by DOE under Contract No. DE-AC02-98CH10886. We thank organisers of 2nd Workshop on the Discovery Potential of a B Factory at $10^{36}$ Luminosity, SLAC (October, 2003) where this work was initiated and G. Burdman, 
Z. Chacko, W. Goldberger, M. Graesser, Y. Grossman, I. Hinchliffe, D. E. Kaplan, Y. Nir, Y. Nomura, F. Petriello, A. Pierce, R. Sundrum, M. Suzuki and J. Wacker for discussions.

\section{References}

[1] L. Randall and R. Sundrum, hep-ph/9905221, Phys. Rev. Lett. 83, 3370 (1999) and hep-th/9906064 Phys. Rev. Lett. 83, 4690 (1999).

[2] J. Maldacena, hep-th/9711200 Adv. Theor. Math. Phys. 2, 231 (1998); S. Gubser, I. Klebanov and A. Polyakov, hep-th/9802109, Phys. Lett. B 428, 105 (1998); E. Witten, hep-th/9802150 Adv. Theor. Math. Phys. 2, 253 (1998); for a review see O. Aharony, S. Gubser, J. Maldacena, H. Ooguri and Y. Oz, hep-th/9905111, Phys. Rept. 323, 183 (2000).

[3] H. Verlinde, hep-th/9906182, Nucl. Phys. B 580, 264 (2000); J. Maldacena, unpublished remarks; E. Witten, ITP Santa Barbara conference 'New Dimensions in Field Theory and String Theory', http://www.itp.ucsb.edu/online/susy_c99/discussion S. Gubser, hep-th/9912001, Phys. Rev. D 63, 084017 (2001); E. Verlinde and H. Verlinde, hep-th/9912018, JHEP 0005, 034 (2000); N. Arkani-Hamed, M. Porrati and L. Randall, hep-th/0012148, JHEP 0108, 017 (2001); R. Rattazzi and A. Zaffaroni, hep-th/0012248, JHEP 0104, 021 (2001); M. Perez-Victoria, hep-th/0105048 JHEP 0105, 064 (2001).

[4] H. Georgi and D. B. Kaplan, Phys. Lett. B 136, 183 (1984) and Phys. Lett. B 145, 216 (1984); S. Dimopoulos, H. Georgi and D. B. Kaplan, Phys. Lett. B 136, 187 (1984); P. Galison, H. Georgi and D. B. Kaplan, Phys. Lett. B 143, 152 (1984); M. J. Dugan, H. Georgi and D. B. Kaplan, Nucl. Phys. B 254, 299 (1985).

[5] T. Gherghetta and A. Pomarol, hep-ph/0003129 Nucl. Phys. B 586, 141 (2000).

[6] S. J. Huber and Q. Shafi, hep-ph/0010195, Phys. Lett. B 498, 256 (2001); S. J. Huber, hep-ph/0303183, Nucl. Phys. B 666, 269 (2003).

[7] Y. Grossman and M. Neubert, hep-ph/9912408, Phys. Lett. B 474, 361 (2000).

[8] S. J. Huber and Q. Shafi, Phys. Rev. D 63, 045010 (2001) arXiv:hep-ph/0005286; S. J. Huber, C. A. Lee and Q. Shafi, Phys. Lett. B 531, 112 (2002) arXiv:hep-ph/0111465; C. Csaki, J. Erlich and J. Terning, Phys. Rev. D 66, 064021 (2002) arXiv:hep-ph/0203034; J. L. Hewett, F. J. Petriello and T. G. Rizzo, JHEP 0209, 030 (2002) arXiv:hep-ph/0203091. 
[9] K. Agashe, A. Delgado, M. J. May and R. Sundrum, hep-ph/0308036 JHEP 0308, 050 (2003).

[10] C. Csaki et al., arXiv:hep-ph/0308038,

[11] R. Barbieri, A. Pomarol and R. Rattazzi, Phys. Lett. B 591, 141 (2004) arXiv:hep-ph/0310285.

[12] H. Davoudiasl et al. arXiv:hep-ph/0312193

[13] G. Burdman and Y. Nomura, arXiv:hep-ph/0312247,

[14] G. Cacciapaglia, C. Csaki, C. Grojean and J. Terning, arXiv:hep-ph/0401160.

[15] R. Barbieri, A. Pomarol, R. Rattazzi and A. Strumia, arXiv:hep-ph/0405040

[16] J. L. Hewett, B. Lillie and T. G. Rizzo, arXiv:hep-ph/0407059

[17] R. Contino, Y. Nomura and A. Pomarol, hep-ph/0306259, Nucl. Phys. B 671148 (2003); K. Agashe, R. Contino and A. Pomarol, to appear.

[18] K. Agashe, G. Perez and A. Soni, arXiv:hep-ph/0406101.

[19] G. Burdman, Phys. Lett. B 590, 86 (2004) arXiv:hep-ph/0310144.

[20] Y. Nomura, JHEP 0311, 050 (2003) arXiv:hep-ph/0309189.

[21] C. Csaki, C. Grojean, J. Hubisz, Y. Shirman and J. Terning, arXiv:hep-ph/0310355.

[22] Y. Nir, arXiv:hep-ph/0109090 Lectures given at 55th Scottish Universities Summer School in Physics: Heavy Flavor Physics, St. Andrews, Scotland, (2001). Published in St. Andrews, Heavy flavour physics 147 (2001).

[23] K. Agashe and A. Delgado, hep-th/0209212, Phys. Rev. D 67, 046003 (2003).

[24] R. Contino and A. Pomarol, arXiv:hep-th/0406257.

[25] E. Witten, Nucl. Phys. B 160, 57 (1979).

[26] G. Buchalla, A. J. Buras and M. E. Lautenbacher, Rev. Mod. Phys. 68, 1125 (1996) arXiv:hep-ph/9512380.

[27] A. F. Falk, Z. Ligeti, Y. Nir and H. Quinn, Phys. Rev. D 69, 011502 (2004) arXiv:hep-ph/0310242.

[28] N. Cabibbo, Phys. Rev. Lett. 10, 531 (1963); M. Kobayashi and T. Maskawa, Prog. Th. Phys. 49, 652 (1973). 
[29] D. Atwood and A. Soni, Phys. Lett. B 508, 17 (2001) arXiv:hep-ph/0103197.

[30] Y. Nir, Nucl. Phys. Proc. Suppl. 117, 111 (2003) arXiv:hep-ph/0208080.

[31] M. Battaglia et al., arXiv:hep-ph/0304132

[32] For the most updated fit see: http://www.slac.stanford.edu/xorg/ckmfitter/ckm_results_winter2004.html

[33] G. Eyal, Y. Nir and G. Perez, JHEP 0008, 028 (2000) arXiv:hep-ph/0008009.

[34] A. Hocker, H. Lacker, S. Laplace and F. Le Diberder, Eur. Phys. J. C 21, 225 (2001) arXiv:hep-ph/0104062;

[35] J. Charles et al. [CKMfitter Group Collaboration], arXiv:hep-ph/0406184

[36] A. Poluektov et al. [Belle Collaboration], arXiv:hep-ex/0406067.

[37] E. Lunghi and D. Wyler, Phys. Lett. B 521, 320 (2001) arXiv:hep-ph/0109149.

[38] D. Atwood and G. Hiller, arXiv:hep-ph/0307251 G. Buchalla, G. Hiller and G. Isidori, Phys. Rev. D 63, 014015 (2001) arXiv:hep-ph/0006136; A. Ali, E. Lunghi, C. Greub and G. Hiller, Phys. Rev. D 66, 034002 (2002) arXiv:hep-ph/0112300; T. Hurth, Rev. Mod. Phys. 75, 1159 (2003) arXiv:hep-ph/0212304; A. Ghinculov, T. Hurth, G. Isidori and Y. P. Yao, Nucl. Phys. B 685, 351 (2004) arXiv:hep-ph/0312128; C. Bobeth, P. Gambino, M. Gorbahn and U. Haisch, JHEP 0404, 071 (2004) arXiv:hep-ph/0312090.

[39] K. Agashe, talk at Second Workshop on the Discovery Potential of an Asymmetric B Factory at $10^{36}$ Luminosity, SLAC, October 22-24, 2003.

[40] A. J. Buras, arXiv:hep-ph/9806471.

[41] A. F. Falk, A. Lewandowski and A. A. Petrov, Phys. Lett. B 505, 107 (2001) arXiv:hep-ph/0012099.

[42] V. V. Anisimovsky et al. [E949 Collaboration], arXiv:hep-ex/0403036.

[43] A. J. Buras, F. Schwab and S. Uhlig, arXiv:hep-ph/0405132

[44] S. H. Kettell, Nucl. Phys. Proc. Suppl. 111, 232 (2002) arXiv:hep-ex/0205029.

[45] A. Soni, Pramana 62, 415 (2004) arXiv:hep-ph/0307107.

[46] A. J. Buras, arXiv:hep-ph/0402191. 
[47] M. Gronau, Y. Grossman, D. Pirjol and A. Ryd, Phys. Rev. Lett. 88, 051802 (2002) arXiv:hep-ph/0107254; Y. Grossman and D. Pirjol, JHEP 0006, 029 (2000) arXiv:hep-ph/0005069.

[48] D. Atwood, M. Gronau and A. Soni, Phys. Rev. Lett. 79, 185 (1997) arXiv:hep-ph/9704272.

[49] B. Aubert et al. [BABAR Collaboration], arXiv:hep-ex/0405082.

[50] J. M. Soares, Nucl. Phys. B 367, 575 (1991);

A. L. Kagan and M. Neubert, Phys. Rev. D 58, 094012 (1998) arXiv:hep-ph/9803368;

K. Kiers, A. Soni and G. H. Wu, Phys. Rev. D 62, 116004 (2000) arXiv:hep-ph/0006280.

[51] M. Nakao, Int. J. Mod. Phys. A 19, 934 (2004) arXiv:hep-ex/0312041.

[52] See, e. g. T. Hurth in [38] and see also [51].

[53] P. G. Harris et al., Phys. Rev. Lett. 82, 904 (1999).

[54] R. D. Peccei, arXiv:hep-ph/9807516 International University School of Nuclear and Particle Physics, Austria (1998).

[55] E. P. Shabalin, Sov. J. Nucl. Phys. 28, 75 (1979); 31, 864 (1980).

[56] T. Banks, Y. Nir and N. Seiberg, arXiv:hep-ph/9403203

[57] V. D. Barger, T. Falk, T. Han, J. Jiang, T. Li and T. Plehn, Phys. Rev. D 64, 056007 (2001) arXiv:hep-ph/0101106; W. Fischler, S. Paban and S. Thomas, Phys. Lett. B 289, 373 (1992) arXiv:hep-ph/9205233.

[58] N. Arkani-Hamed, J. L. Feng, L. J. Hall and H. C. Cheng, Nucl. Phys. B 505, 3 (1997) arXiv:hep-ph/9704205.

[59] G. Burdman and I. Shipsey, Ann. Rev. Nucl. Part. Sci. 53, 431 (2003) arXiv:hep-ph/0310076.

[60] E. Golowich and A. A. Petrov, Phys. Lett. B 427, 172 (1998) arXiv:hep-ph/9802291; A. F. Falk, Y. Grossman, Z. Ligeti and A. A. Petrov, Phys. Rev. D 65, 054034 (2002) arXiv:hep-ph/0110317; A. F. Falk, Y. Grossman, Z. Ligeti, Y. Nir and A. A. Petrov, Phys. Rev. D 69, 114021 (2004) arXiv:hep-ph/0402204.

[61] S. Eidelman et al. [Particle Data Group Collaboration], Phys. Lett. B 592, 1 (2004).

[62] M. Bander, D. Silverman and A. Soni, Phys. Rev. Lett. 43, 242 (1979).

[63] I. Shipsey, arXiv:hep-ex/0207091 
[64] G. Eilam, J. L. Hewett and A. Soni, Phys. Rev. D 44, 1473 (1991)

[Erratum-ibid. D 59, 039901 (1999)].

[65] B. Grzadkowski, J. F. Gunion and P. Krawczyk, Phys. Lett. B 268, 106 (1991).

[66] G. Eilam, J. L. Hewett and A. Soni, Phys. Rev. Lett. 67, 1979 (1991).

[67] D. Atwood, S. Bar-Shalom, G. Eilam and A. Soni, Phys. Rept. 347, 1 (2001) arXiv:hep-ph/0006032.

[68] K. Agashe, G. Perez and A. Soni, work in progress.

[69] D. Atwood, L. Reina and A. Soni, Phys. Rev. D 53, 1199 (1996) arXiv:hep-ph/9506243.

[70] H. Davoudiasl, J. L. Hewett and T. G. Rizzo, hep-ph/0212279 Phys. Rev. D 68, 045002 (2003); M. Carena et al., hep-ph/0212307, Phys. Rev. D 67, 096006 (2003); M. Carena et al. hep-ph/0305188, Phys. Rev. D 68, 035010 (2003); F. del Aguila, M. Perez-Victoria and J. Santiago, hep-th/0302023, JHEP 0302, 051 (2003) and hep-ph/0305119

\section{Appendix}

\section{A Coupling of zero-mode fermion to gauge $\mathrm{KK}$ mode}

The couplings of KK and zero modes are given by overlap of their wavefunctions. Decomposing the $5 D$ gauge fields as $A_{\mu}(x, z)=\sum_{n} A^{(n)}(x) f_{n}(z)$, the wavefunction of gauge KK mode is given by (for simplicity, we omit the index for the three SM gauge groups in the following)

$$
f_{n}(z)=\sqrt{\frac{1}{z_{h}}} \frac{z}{N_{n}}\left[J_{1}\left(m_{n} z\right)+b_{n} Y_{1}\left(m_{n} z\right)\right] .
$$

We can show that it is localized near the TeV brane. We first consider gauge field with $(++)$ boundary condition (in the Higgs models all the SM gauge boson have these boundary conditions), for which the normalization factor, $N_{n}$, is given by

$$
N_{n}^{2}=\frac{1}{2}\left[z_{v}^{2}\left[J_{1}\left(m_{n} z_{v}\right)+b_{n} Y_{1}\left(m_{n} z_{v}\right)\right]^{2}-z_{h}^{2}\left[J_{1}\left(m_{n} z_{h}\right)+b_{n} Y_{1}\left(m_{n} z_{h}\right)\right]^{2}\right] .
$$

The masses of gauge KK modes and $b_{n}$ are found from

$$
\frac{J_{0}\left(m_{n} z_{h}\right)}{Y_{0}\left(m_{n} z_{h}\right)}=\frac{J_{0}\left(m_{n} z_{v}\right)}{Y_{0}\left(m_{n} z_{v}\right)}=-b_{n} .
$$


We will need masses of lightest KK modes only so that henceforth we assume $m_{n} z_{h} \ll 1$. Then, we get $m_{n} z_{v} \approx$ zeroes of $J_{0}+O\left(1 /\left[\log m_{n} z_{h}\right]\right)$. In particular, the mass of the lightest gauge KK mode is given by

$$
m_{1} \approx 2.45 z_{v}
$$

For $m_{n} z_{v} \approx$ zeroes of $J_{0} \gg 1$, i.e., $m_{n} z_{v} \approx \pi(n-1 / 4)$, we find that

$$
N_{n}^{2} \approx \frac{z_{v}}{\pi m_{n}}
$$

As in the case of a flat extra dimension, the zero-mode of gauge field (which is identified with the SM gauge boson) has a flat profile so that its couplings to all particles is given, at tree level, by

$$
g=g_{5 D} / \sqrt{\pi r_{c}}
$$

where $g_{5 D}$ is the $5 D$ gauge coupling. In terms of this $4 D / \mathrm{SM}$ gauge coupling, the coupling of zero-mode fermion to gauge KK mode (in the interaction basis) is given by:

$$
\frac{g^{(n)}(c)}{g}=\sqrt{\pi r_{c}} \int d z \sqrt{-G} \frac{z}{z_{h}} \chi_{0}^{2}(c, z) f_{n}(z),
$$

where $z / z_{h}$ is the funfbein factor and $-G=\left(z / z_{h}\right)^{-5}$ is the determinant of the metric and the wavefunction of the fermion zero-mode is given by

$$
\chi_{0}(c, z)=\sqrt{\frac{1-2 c}{z_{h}\left(e^{k \pi r_{c}(1-2 c)}-1\right)}}\left(\frac{z}{z_{h}}\right)^{2-c} .
$$

Recall that the "canonically" normalized fermion zero-mode wavefucntion is given by $z^{-3 / 2} \chi_{0}(c, z)$. A numerical evaluation of this coupling shows that it has the form of 1st term in Eq. (16). The $\sim$ sign in Eq. (16) stands for an additional $O(1) c$-dependent factor. For a CFT interpretation of this form of the coupling, see section 4

For completeness, we now consider the combination of $U(1)_{R}$ and $U(1)_{B-L}$ which is orthogonal to hypercharge and is denoted by $Z^{\prime}$. It has $(-+)$ boundary condition (i.e., no zero-mode) and the normalization factor for KK modes of $Z^{\prime}$ is given by

$$
N_{n}^{2}=\frac{1}{2}\left[z_{v}^{2}\left[J_{1}\left(m_{n} z_{v}\right)+b_{n} Y_{1}\left(m_{n} z_{v}\right)\right]^{2}-z_{h}^{2}\left[J_{0}\left(m_{n} z_{h}\right)+b_{n} Y_{0}\left(m_{n} z_{h}\right)\right]^{2}\right]
$$

and the masses of gauge KK modes and $b_{n}$ are given by

$$
\frac{J_{1}\left(m_{n} z_{h}\right)}{Y_{1}\left(m_{n} z_{h}\right)}=\frac{J_{0}\left(m_{n} z_{v}\right)}{Y_{0}\left(m_{n} z_{v}\right)}=-b_{n}
$$


so that, for $m_{n} z_{h} \ll 1$, we get $m_{n} z_{v} \approx$ zeroes of $J_{0}$. For $m_{n} z_{v}\left(\approx\right.$ zeroes of $\left.J_{0}\right) \gg 1$, i.e., $m_{n} z_{v} \approx \pi(n-1 / 4)$, we can show that $N_{n}^{2} \approx z_{v} /\left(\pi m_{n}\right)$ as before.

The couplings of fermions to $Z^{\prime} \mathrm{KK}$ mode can be obtained in a similar fashion. The coupling of gauge KK modes to Higgs is obtained by evaluating the wavefunction on $\mathrm{TeV}$ brane (which is (approximately) the same for both $(++)$ and $(-+)$ BC):

$$
\frac{g_{\text {Higgs }}^{(n)}}{g} \approx(-1)^{(n-1)} \sqrt{2 k \pi r_{c}}
$$

\section{B KK fermion wavefunction and spectrum}

Expanding the $5 D$ fermion as $\Psi(x, z)=\sum_{n} \psi^{(n)}(x) \chi_{n}(c, z)$, the wavefunction of KK mode of SM fermion (i.e., $(++)$ boundary condition) with mass $m_{n}$ is given by:

$$
\chi_{n}(c, z)=\left(\frac{z}{z_{h}}\right)^{5 / 2} \frac{1}{N_{n} \sqrt{\pi r_{c}}}\left[J_{\alpha}\left(m_{n} z\right)+b_{\alpha}\left(m_{n}\right) Y_{\alpha}\left(m_{n} z\right)\right],
$$

where $\alpha=|c+1 / 2|$ and $m_{n}$ and $b_{\alpha}$ are given by

$$
\frac{J_{\alpha \mp 1}\left(m_{n} z_{h}\right)}{Y_{\alpha \mp 1}\left(m_{n} z_{h}\right)}=\frac{J_{\alpha \mp 1}\left(m_{n} z_{v}\right)}{Y_{\alpha \mp 1}\left(m_{n} z_{v}\right)} \equiv-b_{\alpha}\left(m_{n}\right),
$$

(with upper (lower) signs for $c>-1 / 2(c<-1 / 2)$ ) and

$$
\begin{aligned}
N_{n}^{2}= & \frac{1}{2 \pi r_{c} z_{h}}\left[z_{v}^{2}\left[J_{\alpha}\left(m_{n} z_{v}\right)+b_{\alpha}\left(m_{n}\right) Y_{\alpha}\left(m_{n} z_{v}\right)\right]^{2}\right. \\
& \left.-z_{h}^{2}\left[J_{\alpha}\left(m_{n} z_{h}\right)+b_{\alpha}\left(m_{n}\right) Y_{\alpha}\left(m_{n} z_{h}\right)\right]^{2}\right] .
\end{aligned}
$$

Just as for gauge KK modes, we will assume below $m_{n} z_{h} \ll 1$ since we are interested in only lightest KK modes. For $-1 / 2<c<1 / 2-\epsilon$ (where $\epsilon \gtrsim 0.1$ ), we get $m_{n} z_{v} \approx$ zeroes of $J_{-c+1 / 2} \approx \pi(n-c / 2)$, where the last formula is valid for $m_{n} z_{v} \gg 1$. Similarly, for $c>1 / 2+\epsilon$, we get $m_{n} z_{v} \approx$ zeroes of $J_{c-1 / 2} \approx \pi(n+c / 2-1 / 2)$, where the last formula is valid for $m_{n} z_{v} \gg 1$.

The wavefunction of KK mode of $u_{R}^{\prime}$ and $d_{R}^{\prime}\left[S U(2)_{R}\right.$ partners of SM $u_{R}$ and $d_{R}$ with $(-+)$ boundary condition] with mass $m_{n}$ is similar to those for $(+,+)$ KK modes, except:

$$
\frac{J_{\alpha}\left(m_{n} z_{h}\right)}{Y_{\alpha}\left(m_{n} z_{h}\right)}=\frac{J_{\alpha \mp 1}\left(m_{n} z_{v}\right)}{Y_{\alpha \mp 1}\left(m_{n} z_{v}\right)}=-b_{\alpha}\left(m_{n}\right) .
$$

and

$$
\begin{aligned}
N_{n}^{2}= & \frac{1}{2 \pi r_{c} z_{h}}\left[z_{v}^{2}\left[J_{\alpha}\left(m_{n} z_{v}\right)+b_{\alpha}\left(m_{n}\right) Y_{\alpha}\left(m_{n} z_{v}\right)\right]^{2}\right. \\
& \left.-z_{h}^{2}\left[J_{\alpha \mp 1}\left(m_{n} z_{h}\right)+b_{\alpha}\left(m_{n}\right) Y_{\alpha \mp 1}\left(m_{n} z_{h}\right)\right]^{2}\right] .
\end{aligned}
$$


For $c>-1 / 2+\epsilon, m_{n} z_{v} \approx$ zeroes of $J_{c-1 / 2} \approx \pi(n-1 / 2+c / 2)$, where the last formula is valid for $m_{n} z_{v} \gg 1$.

We can show that, for both types of KK fermions and for $m_{n} z_{v} \gg 1$

$$
N_{n}^{2} \approx \frac{z_{v}}{z_{h}} \frac{1}{\pi^{2} m_{n} r_{c}}
$$

and that the wavefunctions are localized near the TeV brane (just as for gauge KK mode).

\section{B.1 Couplings of KK fermions to Higgs}

Using eqs. 67 710 one can show that the value of the KK fermion wavefunctions on the $\mathrm{TeV}$ brane is roughly given by $\sqrt{2 k}$ so that

$$
\left.\frac{\text { wavefunction of KK fermion }}{\text { wavefunction of }(\mathrm{SM}) \text { zero-mode fermion }}\right|_{\mathrm{TeV} \text { brane }} \approx f(c)
$$

Hence, in the interaction basis, we get

$$
Q_{i}^{(n)} d_{j}^{(m)} H \text { coupling } \approx 2 \lambda_{5 D i j} k
$$

and similarly for other KK modes. Also,

$$
Q_{i}^{(0)} d_{j}^{(n)} H \text { coupling } \approx \frac{2 \lambda_{5 D i j} k}{f_{d^{i}}}
$$

and similarly other couplings.

These couplings appear in Eq. (17). In particular, this explains $\approx$ sign in Eq. (17), i.e., there is no further $O(1)$ flavor-dependence (cf. coupling to gauge KK mode in Eq. (16)).

\section{B.2 Couplings of KK fermions to gauge KK modes}

The coupling of zero-mode fermion in weak eigenstate basis to $K K$ fermion and gauge $K K$ mode is diagonal in generation space and is given by:

$$
\frac{g^{(0, n, m)}(c)}{g}=\sqrt{\pi r_{c}} \int d z \sqrt{-G} \frac{z}{z_{h}} \chi_{0}(c, z) \chi_{n}(c, z) f_{m}(z)
$$

where, as before, $z / z_{h}$ is the funfbein factor and $-G=\left(z / z_{h}\right)^{-5}$ is the determinant of the metric

Similarly, we can obtain the coupling of 2 KK fermions to gauge KK mode:

$$
\frac{g^{(m, n, p)}(c)}{g}=\sqrt{\pi r_{c}} \int d z \sqrt{-G} \frac{z}{z_{h}} \chi_{m}(c, z) \chi_{n}(c, z) f_{p}(z)
$$

A numerical evaluation of these wavefunction overlaps shows that these couplings have the form of 2 nd and 3rd terms of Eq. (16). Just like for 1st term of Eq. (16) (i.e., coupling of 2 zero-mode fermions to gauge KK mode), the $\sim$ sign in Eq. (16) stands for an additional $O(1) c$-dependent factor (cf. coupling to Higgs in Eq. (17)). For a CFT interpretation of the form of these couplings, see section 4 


\section{B.3 Flavor structure of KK gluon diagram}

Consider the KK gluon contributions to flavor-violating dipole operators, i.e., $C_{7 \gamma}^{\prime}$ and $C_{7 \gamma}$ :

$$
\begin{aligned}
& C_{7 \gamma}^{\prime} \propto v\left[D_{L}^{\dagger} \operatorname{diag}\left(a_{Q^{i}} f_{Q^{i}}^{-1}\right) \lambda_{d 5 D} \operatorname{diag}\left(a_{d^{i}} f_{d^{i}}^{-1}\right) D_{R}\right]_{32} \\
& C_{7 \gamma} \propto v\left[D_{L}^{\dagger} \operatorname{diag}\left(a_{Q^{i}} f_{Q^{i}}^{-1}\right) \lambda_{d 5 D} \operatorname{diag}\left(a_{d^{i}} f_{d^{i}}^{-1}\right) D_{R}\right]_{23},
\end{aligned}
$$

where the above expression is in the special interaction basis in which the bulk masses are diagonal. We have now included flavor dependence parameterized by the $O(1)$ coefficients, $a_{Q^{i}, d^{i}}$, (i.e., flavor-dependence apart from $f^{\prime}$ 's). These appear in the form of the exact couplings of zero-mode fermion to KK fermion and KK gluon in Eq. (16).

Numerical evaluations shows that only the $a_{Q}$ 's have $O(1)$ flavor dependence as follows. It turns out that the KK wavefunctions for down-type quarks are similar since $c_{Q, d}$ 's are all close to $1 / 2$ (KK wavefunctions are not so sensitive to $c^{\prime}$ 's). However, since $c_{Q^{3}} \sim 0.3-0.4$, the wavefunction of zero-mode $b_{L}$ is localized a bit near $\mathrm{TeV}$ brane, whereas $c_{Q^{1,2}} \sim 0.6-0.7$ so that wavefunction of zero-modes are localized near Planck brane. This difference in zero-mode wavefunctions results in $a_{Q^{1,2}} / a_{Q^{3}} \sim 1.5$. Whereas all $c_{d} \approx 0.6-0.7$ so that all zero-modes are localized near Planck brane resulting in very small flavor-dependence in $a_{d}$ 's.

Another effect that might spoil our approximation is splitting in masses between the masses of the same level KK fermions. Using the results of the previous section we find that this splitting is at most of $\mathcal{O}(10 \%)$ for both the doublet and singlet down type quarks and therefore is subdominant for most calculations.

Alltogether we find [up to $\mathcal{O}(10 \%)$ corrections in $a_{Q}$ due to the mild splitting in KK mass]

$$
a_{Q^{i}} \sim O(1) \times(1.5,1.5,1), \quad a_{d^{i}} \sim O(1) \times(0.9,1,1)
$$

Note that $c_{d^{2}} \sim c_{d^{3}}$ (see table 3) so that these two KK masses and hence the last two entries in $a_{d}$ are degenerate.

Thus, the approximation of neglecting flavor dependence in $O(1)$ coefficients in 2nd term of Eq. (16) is very good for the singlet down quarks and is subject for $\mathcal{O}(1)$ corrections for the doublet ones.

Including the above effect, we get

$$
C_{7 \gamma}^{\prime} \propto m_{s}\left(V_{\mathrm{CKM}}\right)_{23}+\mathcal{O}(0.1) \times m_{b}\left(D_{R}\right)_{13}\left(D_{R}\right)_{12},
$$

where 1st (2nd) contribution to $C_{7 \gamma}^{\prime}$ is using left (right)-handed mixing. Thus, NP contribution from KK gluon to $C_{7 \gamma}^{\prime}$ is suppressed by roughly $\left(g_{s} \sqrt{k \pi r_{c}}\right)^{2} M_{W}^{2} / m_{K K}^{2} \sim \mathcal{O}(1 / 10)$ compared to SM contribution to same operator. We conclude that the mild flavor dependence in $a_{d}$ 's does not give a 
significant effect in $b \rightarrow s \gamma$ due to near degeneracy of $c_{d^{2,3}}$ (cf. $b \rightarrow d \gamma$ below). Given eq. (78) one find that the left handed chirality operator also receives NP contributions

$$
C_{7 \gamma}^{\mathrm{RS}} \propto m_{b}\left(V_{\mathrm{CKM}}\right)_{23},
$$

but without enhancment due to large right handed mixing [compare with the leading contribution [44]. Consequently we find that this NP contribution to $C_{7 \gamma}$ is suppressed by $\mathcal{O}(1 / 10)$ compared to SM contributions. We conclude that the $O(1)$ flavor-dependence in $a_{Q}$ does not give interesting effect since mixing is similar to SM, i.e., CKM-like.

Similarly, for $b \rightarrow d$ transition, we get

$$
\begin{aligned}
C_{7 \gamma d}^{\mathrm{RS}} & \propto m_{b}\left(V_{C K M}\right)_{13} \\
C_{7 \gamma d}^{\prime} & \propto \frac{1}{10} m_{b}\left(D_{R}\right)_{13},
\end{aligned}
$$

Consequently the NP contributions to $C_{7 \gamma d}$ are of $\mathcal{O}(10 \%)$ of the size of SM contributions.

In the case of $C_{7 \gamma d}^{\prime}$ the situation is different since the SM contribution are completely negligible. On top of that we know that right-handed mixing are much larger than left-handed. Thus we find that the NP contributions to $C_{7 \gamma d}^{\prime}$ are enhanced even though the dispersion in the $a_{d^{i}}$ is of $\mathcal{O}(10 \%)$.

Plugging the actual values we find the NP contribution are larger than the SM contribution to the same operator by $\mathcal{O}(10)$. It is still only $10 \%$ of the leading SM contributions to $C_{7 \gamma d}$ (Of course, the leading contributions that we find in Eq. (45), are even larger, comparable to the SM leading ones). Note that since the right-handed mixings are larger than left-handed, the contribution to both $C_{7 \gamma}^{\prime}$ and $C_{7 \gamma d}^{\prime}$ would have been comparable to SM contributions to $C_{7 \gamma}$ and $C_{7 \gamma d}^{\prime}$, respectively if the $a_{d}$ 's had $\mathcal{O}(1)$ flavor dependence. This is exactly the case in the up quark sector which we consider in the following.

There is a new effect in up-quark sector as follows. In addition to $O(1)$ flavor-dependence in $a_{Q}$ (same as down sector), there is $O(1)$

flavor-dependence in $a_{u}$. This implies that approximation of neglecting the flavor dependence in $\mathcal{O}(1)$ coefficients in the 2nd term of Eq. (16) is not valid for both left and right-handed up quarks. This is because $c_{u^{3}} \lesssim 0$, i.e., much smaller than $c_{u^{1,2}}$ which are $\approx 0.6-0.7$. Thus, the zero-mode wavefunction of $t_{R}$ is localized near $\mathrm{TeV}$ brane, whereas $u_{R}$ and $c_{R}$ are localized near Planck brane. In addition, the wavefunction and spectrum of $K K$ modes is different for $t_{R}$ as compared to $u_{R}$ and $c_{R}$ (cf. down type quarks). Both these effects result in $O(1)$ flavor dependence in $a_{u}$. Thus, NP contributions to both $t_{R} \rightarrow c_{L} \gamma$ and $t_{L} \rightarrow c_{R} \gamma$ from KK gluon exchange are comparable to those from Higgs diagrams (unlike for down-type quarks). 


\section{Higgs contributions to dipole operators}

The flavor structure of Higgs contributions to the dipole operators [discussed in subsection [5.3)] are of two types. One is mediated through both down and up type Yukwa couplings while the other proceeds only through down type Yukawa couplings. Below we calculate the flavor structure of the induced operators

$$
O_{\gamma}^{i j}=\bar{d}_{L}^{i} \sigma_{\mu \nu} d_{R}^{j} F^{\mu \nu}
$$

where $F^{\mu \nu}$ is the field strength for the electromagnetic (or strong) interaction and $i, j=1 . .3$. The Wilson coefficient of the corrresponding NP contribution, $C_{\gamma}^{i j}\left(\right.$ or $C_{8 g}^{i j}$ ), are proportional to

$$
C_{\gamma}^{i j} \propto 2 k^{3} v F_{Q}\left(\lambda_{u 5 D} \lambda_{u 5 D}^{\dagger}+\lambda_{d 5 D} \lambda_{d 5 D}^{\dagger}\right) \lambda_{d 5 D} F_{d}
$$

where the above is in the interaction basis. ${ }^{19}$ Before we make a detailed computation we can check whether the above two contribution can in principle yield non-trivial flavor physics. This is the case if the two contrbutions are separately missaligned with the down type mass matrix $F_{Q} \lambda_{d 5 D} F_{d}$. In order to check whether they are aligned we calculated the corresponding commutators and found that, in general, the commutators are non zero so that $C_{\gamma}^{i j}$ and $4 D$ down-type Yukawa cannot be diagonalized simultanously.

\section{C.1 Dipole operators - generic structure}

In order to compute the size of the new contribution to $C_{\gamma}^{i j}$ we manipulate the above expression and rewrite it as a function of SM fermion masses (in the mass basis):

$$
\begin{aligned}
C_{\gamma}^{i j} \propto & 2 k^{3} v\left[F_{Q}\left(\lambda_{u 5 D} \lambda_{u 5 D}^{\dagger}+\lambda_{d 5 D} \lambda_{d 5 D}^{\dagger}\right) \lambda_{d 5 D} F_{d}\right]_{i j} \\
= & 2 k^{3} v\left[F_{Q}\left(\lambda_{u 5 D} \lambda_{u 5 D}^{\dagger}+\lambda_{d 5 D} \lambda_{d 5 D}^{\dagger}\right) F_{Q}^{-1} F_{Q} \lambda_{d 5 D} F_{d}\right]_{i j} \\
= & k^{2} m_{d^{j}}\left[F_{Q}\left(\lambda_{u 5 D} \lambda_{u 5 D}^{\dagger}+\lambda_{d 5 D} \lambda_{d 5 D}^{\dagger}\right) F_{Q}^{-1}\right]_{i j} \\
= & k^{2} m_{d^{j}}\left[\left(F_{Q} \lambda_{u 5 D} F_{u} F_{u}^{-1} \lambda_{u 5 D}^{\dagger}\right.\right. \\
& \left.\left.\quad+F_{Q} \lambda_{d 5 D} F_{d} F_{d}^{-1} \lambda_{d 5 D}^{\dagger}\right) F_{Q}^{-1}\right]_{i j} \\
= & \frac{m_{d^{j}}}{4 v^{2}}\left\{\left[V_{\mathrm{CKM}}^{\dagger} \operatorname{diag}\left(m_{u, c, t}\right) U_{R}^{\dagger} \operatorname{diag}\left(f_{u^{i}}^{2}\right) U_{R} \operatorname{diag}\left(m_{u, c, t}\right) U_{L}^{\dagger}\right.\right. \\
& \left.\left.\quad+m_{d^{i}} D_{R}^{\dagger} \operatorname{diag}\left(f_{d^{i}}^{2}\right) D_{R} \operatorname{diag}\left(m_{d, s, b}\right) D_{L}^{\dagger}\right] \operatorname{diag}\left(f_{Q^{i}}^{2}\right) D_{L}\right\}_{i j},
\end{aligned}
$$

\footnotetext{
${ }^{19}$ In the Higgs diagram with up-type $5 D$ Yukawa, there is additional flavor-violation from splitting in KK spectrum for $u$ due to $c_{u^{3}}<<c_{u^{1,2}}$. Whereas, $d, Q$ KK modes are almost degenerate (due to all $c$ 's $\sim 1 / 2$ ) so that there is no such effect in Higgs diagram with only down-type $5 D$ Yukawa or in the KK gluon diagram for down-type quarks as already mentioned.
} 
where the first (second) term in each equality comes from the diagram in fig. 6 (fig. 5) and involve both up and down (only down) KK quarks and in the last line we give the explicit form of $F_{Q} F_{Q}^{\dagger}$ and $F_{u, d}^{\dagger} F_{u, d}$ in the mass basis. Without loss of generality one can always go to a basis in which $F_{Q, u, d}$ are real and diagonal. In this basis the down type contibutions, $C_{\gamma}^{d^{\mathrm{KK}}}$, are proportional to:

$$
\left(C_{\gamma}^{d^{\mathrm{KK}}}\right)_{i j} \propto \frac{m_{d^{j}} m_{d^{i}}}{4 v^{2}}\left[R \operatorname{diag}\left(m_{d, s, b}\right) L\right]_{i j},
$$

where

$$
(R, L)_{i j}=\sum_{n}\left(D_{R, L}^{*}\right)_{n i}\left(D_{R, L}\right)_{n j} f_{d^{n}, Q^{n}}^{2} .
$$

One can convince himself that order of magnitude-wise

$$
(L, R)_{i j} \sim\left(D_{R, L}^{*}\right)_{1 i}\left(D_{R, L}\right)_{1 j} f_{d^{1}, Q^{1}}^{2} .
$$

Thus the elements of $R, L$ has the following hierarchy,

$$
\begin{aligned}
\frac{(L, R)_{12}}{(L, R)_{11}} \sim \frac{f_{Q^{2}, d^{2}}}{f_{Q^{1}, d^{1}}}, \quad \frac{(L, R)_{22}}{(L, R)_{11}} \sim \frac{f_{Q^{2}, d^{2}}^{2}}{f_{Q^{1}, d^{1}}^{2}}, \quad \frac{(L, R)_{13}}{(L, R)_{11}} \sim \frac{f_{Q^{3}, d^{3}}}{f_{Q^{1}, d^{1}}}, \\
\frac{(L, R)_{23}}{(L, R)_{11}} \sim \frac{f_{Q^{2}, d^{2}} f_{Q^{3}, d^{3}}}{f_{Q^{1}, d^{1}}^{2}}, \quad \frac{(L, R)_{33}}{(L, R)_{11}} \sim \frac{f_{Q^{3}, d^{3}}^{2}}{f_{Q^{1}, d^{1}}^{2}} .
\end{aligned}
$$

The up type contibutions, $C_{\gamma}^{u^{\mathrm{KK}}}$, are proportional to:

$$
\left(C_{\gamma}^{u^{\mathrm{KK}}}\right)_{i j} \propto \frac{m_{d^{j}}}{4 v^{2}}\left[V_{\mathrm{CKM}}^{\dagger} \operatorname{diag}\left(m_{u, c, t}\right) \bar{R} \operatorname{diag}\left(m_{u, c, t}\right) \bar{L} V_{\mathrm{CKM}}\right]_{i j},
$$

where

$$
(\bar{R}, \bar{L})_{i j}=\sum_{n}\left(U_{R, L}^{*}\right)_{n i}\left(U_{R, L}\right)_{n j} f_{u^{n}, Q^{n}}^{2},
$$

and magnitude-wise we find

$$
(\bar{L}, \bar{R})_{i j} \sim\left(U_{R, L}^{*}\right)_{1 i}\left(U_{R, L}\right)_{1 j} f_{u^{1}, Q^{1}}^{2},
$$

and the pattern of hierarchy between the elements of $\bar{L}, \bar{R}$ is similar to what shown in 866.

\section{C.2 Dipole operators and $b \rightarrow d, s \gamma$}

Using eqs. 8487) we can estimate, in term of spurions, what is the NP contribution, $C_{\gamma}^{\lambda_{d}}, C_{\gamma}^{\lambda_{u}}$, to the opposite chiralty operator $C_{7 \gamma}^{\prime}$.

We first consider the contribution from the diagram in fig. 5 which contains only down type KK quark. The relevant NP contribution to $b \rightarrow s \gamma$ is 
proportional $\left(C_{\gamma}^{d^{\mathrm{KK}}}\right)_{32}$ (84),

$$
\begin{aligned}
C_{7 \gamma}^{\lambda_{d}} & \propto\left(C_{\gamma}^{d^{\mathrm{KK}}}\right)_{32}=\frac{m_{s} m_{b}}{4 v^{2}}\left[R \operatorname{diag}\left(m_{d, s, b}\right) L\right]_{32} \\
& \sim \frac{m_{s} m_{b}}{4 v^{2}}\left(m_{d} R_{31} L_{12}+m_{s} R_{32} L_{22}+m_{b} R_{33} L_{32}\right) \\
& \sim \frac{m_{s} m_{b}}{4 v^{2}} f_{d^{3}} f_{Q^{2}}\left(a_{1} m_{d} f_{Q^{1}} f_{d^{1}}+a_{2} m_{s} f_{d^{2}} f_{Q^{2}}+a_{3} m_{b} f_{d^{3}} f_{Q^{3}}\right) \\
& \sim a_{d} \frac{m_{s} m_{b}}{4 v^{2}} f_{Q^{2}} f_{d^{2}}\left(D_{R}\right)_{23} 2 k v \lambda_{5 D} \sim\left(\lambda_{5 D} k\right)^{2} m_{b}\left(D_{R}\right)_{23}
\end{aligned}
$$

where $a_{i, d}$ is an order one complex number. Similarly the contribution from the diagram in fig. 6 which contains both down and up type KK quarks is proportional $\left(C_{\gamma}^{u^{\mathrm{KK}}}\right)_{32}$ [see eq. (87)],

$$
\begin{aligned}
C_{7 \gamma}^{\lambda_{u}} & =\left(C_{\gamma}^{u^{\mathrm{KK}}}\right)_{32} \propto \frac{m_{s}}{4 v^{2}}\left[V_{\mathrm{CKM}}^{\dagger} \operatorname{diag}\left(m_{u, c, t}\right) \bar{R} \operatorname{diag}\left(m_{u, c, t}\right) \bar{L} V_{\mathrm{CKM}}\right]_{32} \\
& \sim a_{u} \frac{m_{s} m_{t} m_{u}}{4 v^{2}} f_{u^{3}} f_{Q^{2}} f_{Q^{1}} f_{u^{1}} \sim a_{u} \frac{m_{s} m_{t}}{2 v} k \lambda_{5 D} f_{u^{3}} f_{Q^{2}} \\
& \sim a_{u} m_{s}\left(k \lambda_{5 D}\right)^{2} \frac{f_{Q^{2}}}{f_{Q^{1}}} \sim\left(\lambda_{5 D} k\right)^{2} m_{b}\left(D_{R}\right)_{23},
\end{aligned}
$$

where $a_{u}$ is an order one complex number and in this case there are nine terms of similar order so for simplicitly we represented them by a single term with a coefficient $a_{u}$.

Similar derivation yields the NP contributions to the dipole moment operator $O_{7 \gamma d}^{\prime}$ which mediates the $b \rightarrow d \gamma$ process:

$$
C_{7 \gamma d}^{\prime} \propto\left(\lambda_{5 D} k\right)^{2} m_{b}\left(D_{R}\right)_{13}
$$

\section{C.3 Flavor diagonal dipole operators}

In the above we showed explicitly that the above framework yields sizable contribution to the dipole operators $O_{7 \gamma}^{\prime}, O_{7 \gamma}^{\prime d}$. In this part we ask whether similar contributions may yield also contribution to flavor diagonal CPV observable of the forms of neutron and electron EDMs.

In order to answer the above question we need to calculate the relevant Wilson coefficient, $C_{d_{n}}$, that is generated by the diagram in figures 5 and 6 (with $d_{L, R}$ external quarks). We aim to demonstrate that a physical imaginary part of $C_{d_{n}}$ is generated from the new diagrams. We shall see that the contribution we get is due to the presence of the "Majorana-like" phases. In particular we find below that it is enough to have two generations in order to obtain the non-vanishing contributions. For simplicity, to demonstrate our point, it is enough to consider only the contribution with internal down quarks (figure 5). The diagram in figure 6 is expected to induce similar but independent contributions of roughly the same magnitude. We start by using the result for 
$C_{\gamma}^{i j}$ (83) with $i, j=1$, :

$$
C_{d_{n}} \propto \frac{m_{d}^{2}}{4 v^{2}}\left[\left(F_{d}^{\dagger} F_{d}\right)^{-1}\left(m_{d, s, b}\right)\left(F_{Q} F_{Q}^{\dagger}\right)^{-1}\right]_{11},
$$

where the above result is in the mass basis. It is clear that the above Wilson coefficient receive non-zero contributions. In order to have CPV, however, we should check whether the above contributions contains non removable CP phases, i.e, physical CPV phases. Since the external quarks are in the mass basis the only phase redefinition freedom allowed is a vector like rotation, $d_{L, R}^{i} \rightarrow d_{L, R}^{i} e^{i \chi^{i}}$. One can easily convince himself that this transformation leaves invariant the 11 element of the object in the square paranethesis of (92). Consequently $C_{d_{n}}$ cannot be brought to be real by such a simple field redefinition.

In order to explicitly calculate the imaginary part of $C_{d_{n}}$ we look more closely at the expression in (92).

$$
C_{d^{n}}^{1} \propto\left[D_{R}^{\dagger} \operatorname{diag}\left(f_{d^{1}, d^{2}, d^{3}}^{2}\right) D_{R} \operatorname{diag}\left(m_{d, s, b}\right) D_{L}^{\dagger} \operatorname{diag}\left(f_{Q^{1}, Q^{2}, Q^{3}}^{2}\right) D_{L}\right]_{11},
$$

where this is in the "special basis" in which $F_{Q, d}$ are real and diagonal.

Let us make a short detour from the main route of the above discussion to see how this fits with our discussion in subsection (3.3.2). In that part we showed that if we have flavor violation only in the down type sector then $D_{L, R}$ contains $4 \mathrm{CPV}$ phases. In order to see how these are distributed consider, e.g., the following general parameterization of a $3 \times 3$ complex down quark mass matrix, $M_{d}$,

$$
M_{d}=D_{L} \operatorname{diag}\left(m_{d, s, b}\right) D_{R}^{\dagger},
$$

where $D_{L, R}$ are $3 \times 3$ unitary matrices:

$$
\begin{aligned}
D_{R}^{\dagger} & =P_{D} R_{12}^{R} R_{13}^{R} \operatorname{diag}\left(1,1, e^{i \delta^{R}}\right) R_{23}^{R} \operatorname{diag}\left(1, e^{i \theta_{1}^{R}}, e^{i \theta_{2}^{R}}\right), \\
D_{L}^{\dagger} & =R_{12}^{L} R_{13}^{L} \operatorname{diag}\left(1,1, e^{i \delta^{L}}\right) R_{23}^{L} \operatorname{diag}\left(1, e^{i \theta_{1}^{L}}, e^{i \theta_{2}^{L}}\right),
\end{aligned}
$$

where $P_{D}=\operatorname{diag}\left(e^{i \phi_{1}}, e^{i \phi_{2}}, e^{i \phi 3}\right)$ and $R_{i j}^{L, R}$ stands for an $\mathrm{SO}(3)$ matrix which describes a rotation in the $i j$ plane and $\delta_{R, L}$ are CKM like phases while the other are "Majorana" like. ${ }^{20}$ As discussed in subsection 3.3 .2 we have, still in the "special", interaction, basis, a freedom to rotate five phases using field redefinitions for the down quarks in the interaction basis $d_{L, R}^{i} \rightarrow d_{L, R}^{i} e^{i \chi_{L, R}^{i}}$ (in order to remain in the "special basis", in which $F_{Q, u, d}$ are real and diagonal, all the KK excitation should also be similarly rotated). This will modify the form of the mass matrix which will contain only 4 phases $M_{d} \rightarrow \tilde{M}_{d}=\tilde{D}_{L} \operatorname{diag}\left(m_{d, s, b}\right) \tilde{D}_{R}^{\dagger}$, where

$$
\begin{aligned}
& \tilde{D}_{R}^{\dagger}=\operatorname{diag}\left(1, e^{i \tilde{\phi}_{1}}, e^{i \tilde{\phi}_{2}}\right) R_{12}^{R} \operatorname{diag}\left(1,1, e^{i \delta^{R}}\right) R_{13}^{R} R_{23}^{R}, \\
& \tilde{D}_{L}^{\dagger}=R_{12}^{L} \operatorname{diag}\left(1,1, e^{i \delta^{L}}\right) R_{13}^{L} R_{23}^{L},
\end{aligned}
$$

${ }^{20}$ Note that the above mass matrix has 9 idependent phases as required. 
where in this definition only $\tilde{D}_{R}$ contains, unremovable, Majorana type phases $\tilde{\phi}_{1,2}$. These can however be shifted to $\tilde{D}_{L}$ using vector like field redefinitions in the mass basis. Thus, with mixing, the product in Eq. (93) might have non zero imaginary part which appears when several flavors are involved in a physical process (since the contribution of only $\left(D_{R, L}\right)_{11}$ in Eq. (93) is real). Let us write the above expression more explicitly:

$$
\begin{aligned}
C_{d^{n}}^{1} \propto \quad & \left(R_{11}, \frac{m_{s}}{m_{d}} R_{12}, \frac{m_{b}}{m_{d}} R_{13}\right) \\
& \cdot\left(L_{11}, L_{12}, L_{13}\right)^{T},
\end{aligned}
$$

where

$$
(R, L)_{i j}=\sum_{n}\left(D_{R, L}^{*}\right)_{n i}\left(D_{R, L}\right)_{n j} f_{d^{n}, Q^{n}}^{2}
$$

and the dot stands for a scalar product between the two vectors. Note the in the above expression the first element of each vector is real thus cannot contribute to the EDM.

To further simplify the analysis of (97) we move to a two generations framework. In that case we have a single, unremovable, CPV "Majorana" like phase which again can be shifted from $D_{L}$ to $D_{R}$

$$
\tilde{D}_{R}^{\dagger}=\operatorname{diag}\left(1, e^{i \tilde{\phi}}\right) R_{12}^{R}, \quad \tilde{D}_{L}^{\dagger}=R_{12}^{L},
$$

Then we find

$$
C_{d^{n}}^{1} \propto R_{11} L_{11}+\frac{m_{s}}{m_{d}}\left[e^{-i \tilde{\phi}} f_{1}^{2}\left|\left(D_{R}\right)_{11}\left(D_{R}\right)_{12}\right|+f_{2}^{2}\left(D_{R}\right)_{21}\left(D_{R}\right)_{22}\right] L_{12},
$$

where note that the above expression is invariant with under vector-like field redefinitions in the mass basis. Thus the above does contribute to the EDM. Furthermore using eq. (86) one can convince himself that the contribution is unsuppressed since the suppression due to mixing is compensated by the $m_{s} / m_{d}$ enhancement so that altogether we find

$$
\operatorname{Im}\left(C_{d^{n}}^{1}\right) \propto m_{d} .
$$

It is clear that also in the three generation case a similar result is obtained. This is since the contribution is due to the non-removable Majorana phases and we showed in the above that the resultant structure is invariant with respect to field redefinitions. 